\title{
Convergence of an adaptive discontinuous Galerkin method for elliptic interface problems
}

\author{
Andrea Cangiani $^{\mathrm{a}}$, Emmanuil H. Georgoulis ${ }^{\mathrm{b}}$, Younis Sabawi ${ }^{\mathrm{c}}$ \\ ${ }^{a}$ School of Mathematical Sciences, University of Nottingham, University Park, Nottingham, NG7 2RD, United \\ Kingdom, United Kingdom (e-mail: Andrea.Cangiani@nottingham.ac.uk). \\ ${ }^{b}$ Department of Mathematics, University of Leicester, University Road, Leicester, LE1 7RH, United Kingdom, and \\ Department of Mathematics, School of Applied Mathematical and Physical Sciences, National Technical University of \\ Athens, Zografou 157 80, Greece (e-mail: Emmanuil.Georgoulis@le.ac.uk) \\ ${ }^{c}$ Department of Mathematics, Faculty of Science and Health, Koya University, Koya KOY45, Kurdistan Region - F. R. \\ Iraq, and Department of Mathematics Education, Faculty of Education, Tishk International University, Kurdistan-Iraq \\ (e-mail: younis.abid@koyauniversity.org,younis.sabawi@ishik.edu.iq)
}

\begin{abstract}
We prove a basic error contraction result of an adaptive discontinuous Galerkin method for an elliptic interface problem. The interface conditions considered model mass transfer of solutes through semipermeable membranes and other filtering processes. The adaptive algorithm is based on a residualtype a posteriori error estimator, with a bulk refinement criterion. The a posteriori error bound is derived under the assumption that the triangulation is aligned with the interfaces although, crucially, extremely general curved element shapes are also allowed, resolving the interface geometry exactly. As a corollary, convergence of the adaptive discontinuous Galerkin method for non-essential Neumannand/or Robin-type boundary conditions, posed on general curved boundaries, also follows. Numerical experiments are also presented.
\end{abstract}

Keywords: Discontinuous Galerkin method, interface problem, a posteriori error bound, adaptivity, convergence analysis, a posteriori error analysis on curved domains.

\section{Introduction}

Initial- and boundary-value problems posed on multi-compartment geometries closed by interface conditions are abundant in physical and biological modelling as well as in respective (bio-)engineering applications. A number of finite element-type methods to approximate solutions to such problems, especially in the context of general, possibly curved, interfaces have been proposed over the years, such as the unfitted FEM [19], immersed interface methods [24, 25, 29], fictitious domain methods $[3,5,6]$, composite FEM [30], cut-cell techniques [26, 31, 18], and nonconforming and discontinuous Galerkin FEM in [8, 7]. Many of the above works provide a priori error analysis of the proposed methods and/or error estimation using adjoint techniques.

An a posteriori error analysis for a fitted discontinuous Galerkin $(\mathrm{dG})$ method for elliptic interface problems with mass-flux balance interface conditions modelling, e.g., mass transfer of solutes through semi-permeable membranes, based on residual-type a posteriori error estimators, has been established in [13]. However, the issue of convergence was not addressed. A key attribute in the analysis presented in [13] was the ability to use elements with very general curved faces allowing for "fitted" approximation of essentially arbitrary interface geometries. This is in sharp contrast with various results involving a posteriori error bounds for finite element methods treating classical interface problems, whereby the interface geometry is dictated by the mesh skeleton which is typically a piecewise linear (or, at most polynomial) curve/surface; see, e.g., [32].

Whilst the topic of the convergence analysis of adaptive algorithms for elliptic problems is now relatively well understood for both conforming and non-conforming methods, see, e.g., [27, 23, 14, 15, $4,20,21,28]$ for works related in spirit to the developments in the present works, to the best of our knowledge, no results in the context of elliptic interface problems exist. 


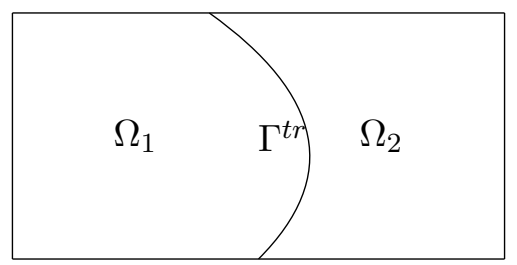

Figure 1: The computational domain $\Omega$ consisting of two disjoint sub-domains $\Omega_{1}$ and $\Omega_{2}$, separated by the interface $\Gamma^{t r}=\bar{\Omega}_{1} \cap \bar{\Omega}_{2}$.

In this work, we continue the study of the adaptive $\mathrm{dG}$ method from [13] by proving an error contraction result for a simple adaptive algorithm in the spirit of $[14,4]$, subject to the use of a bulk refinement criterion. The mass-flux balance condition posed over a curved interface, and discretised using general curved elements, poses a series of new challenges compared to the respective results of $[23,4]$. A discussion of admissible meshes possibly comprising of general curved elements in the neighbourhood of the interface is also given. We refrain from discussing a respective quasi-optimality result, as the function-space framework for this class of problems is not entirely settled, to the best of our knowledge and, therefore, no respective embeddings of such interface-type approximation classes to standard Sobolev-Besov-type spaces are available. Remarkably, upon restricting the analysis to one compartment geometry, our result also shows convergence of the fitted adaptive dG method for (one-compartment) boundary-value problems with non essential boundary conditions of Neumann or Robin type posed on general piecewise curved geometries.

The remainder of this work is organised as follows. In Sections 2 and 3, the model problem and the discontinuous Galerkin method along with the admissible curved element shapes, are discussed. Some necessary approximation, trace, and inverse estimates for general curved elements are presented in Section 4. In Sections 4.2 and 5, an extension of the conforming-nonconforming recovery operator from [22] to curvilinear elements, proven in [13] is recalled, along with the upper and lower a posteriori bounds for the dG method. The contraction result is proven in Section 6. Finally, some numerical experiments investigating the performance of the a posteriori error bounds are presented in Section 7 .

\section{Model problem}

Let $\Omega$ be a bounded open polygonal/polyhedral domain with Lipschitz boundary $\partial \Omega$ in $\mathbb{R}^{d}, d=2,3$. $\Omega$ is split into two sub-domains $\Omega_{1}$ and $\Omega_{2}$, such that $\Omega=\Omega_{1} \cup \Omega_{2} \cup \Gamma^{t r}$, with $\Gamma^{t r}:=\bar{\Omega}_{1} \cap \bar{\Omega}_{2}$ being also Lipschitz continuous with bounded curvature, with $\bar{W} \subset \mathbb{R}^{d}$ denoting the closure of a set under that standard Euclidean distance; see Figure 1 for an illustration. We consider the model problem:

$$
\begin{aligned}
-\Delta u & =f, & & \text { in } \Omega_{1} \cup \Omega_{2}, \\
u & =0, & & \text { on } \partial \Omega, \\
\mathbf{n}^{1} \cdot \nabla u_{1} & =\left.C_{t r}\left(u_{2}-u_{1}\right)\right|_{\Omega_{1}} & & \text { on } \bar{\Omega}_{1} \cap \Gamma^{t r}, \\
\mathbf{n}^{2} \cdot \nabla u_{2} & =\left.C_{t r}\left(u_{1}-u_{2}\right)\right|_{\Omega_{2}} & & \text { on } \bar{\Omega}_{2} \cap \Gamma^{t r},
\end{aligned}
$$

with $u_{i}=\left.u\right|_{\bar{\Omega}_{i}}, i=1,2, C_{t r}>0$ a given interface transmission (e.g., permeability) constant and $\mathbf{n}^{i}$, $i=1,2$ denoting the respective outward unit normal vectors. This is a simplified model for mass transfer of a solute through a semi-permeable membrane through osmosis, see eg. [9, 10, 12], but it is rich enough in highlighting the aforementioned challenges posed for the numerical analysis of this class of problems. Also, we set $\mathcal{H}^{1}:=H^{1}\left(\Omega_{1} \cup \Omega_{2}\right)$, and

$$
\mathcal{H}_{0}^{1}:=\left\{v \in \mathcal{H}^{1}: v=0 \text { on } \partial \Omega\right\} .
$$

Upon integrating by parts on each sub-domain and applying the interface condition, we arrive to (1) in weak form, reading: find $u \in \mathcal{H}_{0}^{1}$ such that

$$
D(u, v):=\int_{\Omega_{1} \cup \Omega_{2}} \nabla u \cdot \nabla v d x+\int_{\Gamma^{t r}} C_{t r} \llbracket u \rrbracket \cdot \llbracket v \rrbracket d s=\int_{\Omega} f v d x,
$$


for all $v \in \mathcal{H}_{0}^{1}$, where $\llbracket u \rrbracket:=\left.v_{1}\right|_{K} \mathbf{n}^{1}+\left.v_{2}\right|_{K} \mathbf{n}^{2}$ is the jump across the interface.

\section{The discontinuous Galerkin method}

In this section, we recall from [13] the mesh setting and $d G$ method for problem (2).

\subsection{The mesh}

Let $\mathcal{T}=\{K\}$ be a locally quasi-uniform subdivision of $\Omega$, possibly containing regular hanging nodes, with $K$ a generic, possibly curved, simplicial, box-type, or prismatic element. More specifically, we shall assume that the mesh consists of triangular or quadrilateral elements when $d=2$, and of tetrahedral or prismatic elements with triangular bases when $d=3$. We stress that the prismatic elements considered here are not assumed to have parallel bases, in general.

The mesh skeleton $\Gamma:=\cup_{K \in \mathcal{T}} \partial K$ is subdivided into three disjoint subsets $\Gamma=\partial \Omega \cup \Gamma^{i n t} \cup \Gamma^{t r}$, where $\Gamma^{i n t}:=\Gamma \backslash\left(\partial \Omega \cup \Gamma^{t r}\right)$. We make some further assumptions on the mesh in the vicinity of the interface $\Gamma^{t r}$. In particular, we assume that each element $K \in \mathcal{T}$ such that $\partial K \cap \Gamma^{t r} \neq \emptyset$ has exactly one whole face $E \subset \Gamma^{t r}$; this implies that each such element has all the vertices of the face $E \subset \Gamma^{t r}$ lying on $\Gamma^{t r}$. Moreover, we assume that the mesh is constructed in such a way that each element $K$ is a Lipschitz domain.

We shall assume that curved elements are employed only to resolve the interface geometry, i.e., only elements $K \in \mathcal{T}$ such that $\partial K \cap \Gamma^{t r} \neq \emptyset$ are curved, see Figure 2 for an illustration.

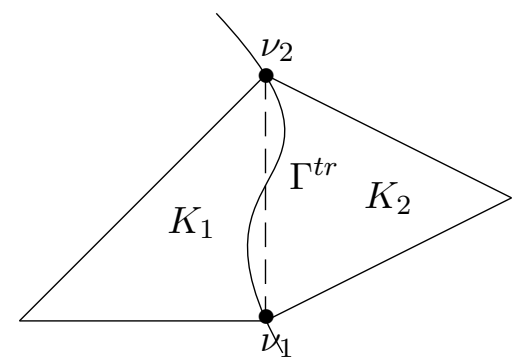

Figure 2: Curved elements $K_{1}$ and $K_{2}$ (solid lines/curves) from either side of the interface $\Gamma^{t r}$, resolving the geometry of $\Gamma^{t r}$.

Moreover, we assume that no interior point of an element $K \in \mathcal{T}$ (which we recall is an open set) can have a non-trivial intersection with the interface $\Gamma^{t r}$. Furthermore, for simplicity and with no essential loss of generality, we assume that the set $\partial K \cap \Gamma^{t r} \neq \emptyset$ is one whole face of $K$, or one vertex of $K$ only. Hence, when $d=3$, we shall only consider (possibly curved) tetrahedral or prismatic elements with triangular bases $K \in \mathcal{T}$ such that $\partial K \cap \Gamma^{t r} \neq \emptyset$, so that a unique cut plane passes through the 3 vertices of $K$ lying on $\Gamma^{t r}$. We collect such interface elements in the local set

$$
\mathcal{T}^{t r}:=\left\{K \in \mathcal{T}: \operatorname{meas}_{d-1}\left(\partial K \cap \Gamma^{t r}\right)>0\right\},
$$

where $\operatorname{meas}_{r}(\omega)$ denotes the $r$-dimensional Hausdorff measure of a set $\omega \subset \mathbb{R}^{d}$; see Figure 2 for an illustration of such elements. Note that elements having just one vertex on $\Gamma^{t r}$ do not belong to $\mathcal{T}^{t r}$.

Assumption 3.1 We assume that:

a) (star-shapedness) each element $K \in \mathcal{T}^{\text {tr }}$, having the face $E \subset \Gamma^{\text {tr }}$, is star-shaped with respect to all vertices opposite this face $E$; note that we have one such vertex when $K$ is simplicial, or more than one such vertices when $K$ is box-type or prismatic. Furthermore, we assume that each element $K \in \mathcal{T}^{\text {tr }}$ is also star-shaped with respect to all the midpoints of the edges sharing a common vertex with the face $E \subset \Gamma^{\text {tr }}$ and are not (edges of) $E \subset \Gamma^{\text {tr }}$ itself; we refer to Figure 3 for an illustration for $d=2$.

b) (shape-regularity) we have $\mathbf{m}(\mathbf{x}) \cdot \mathbf{n}(\mathbf{x}) \geq c|\mathbf{m}(\mathbf{x})|$ uniformly across the mesh, for every vector $\mathbf{m}(\mathbf{x})=\mathbf{x}-\mathbf{x}_{\mathbf{0}}$, with $\mathbf{x} \in E$ and $\mathbf{x}_{0}$ any vertex opposite $E \in \Gamma$, and $\mathbf{n}(\mathbf{x})$ the respective unit outward normal vector to $E$ at $\mathbf{x}$. Moreover, we assume that $|\mathbf{m}(\mathbf{x})| \sim h_{K}$ uniformly. 

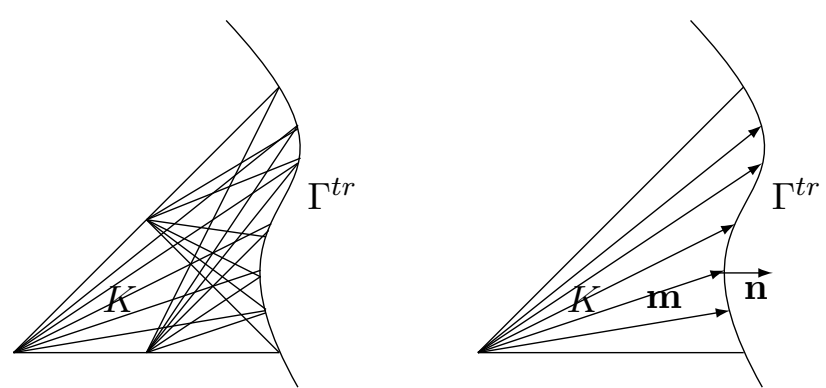

Figure 3: Elements $K \in \mathcal{T}^{t r}$, are assumed to satisfy Assumption 3.1 a) (left) and b) (right)

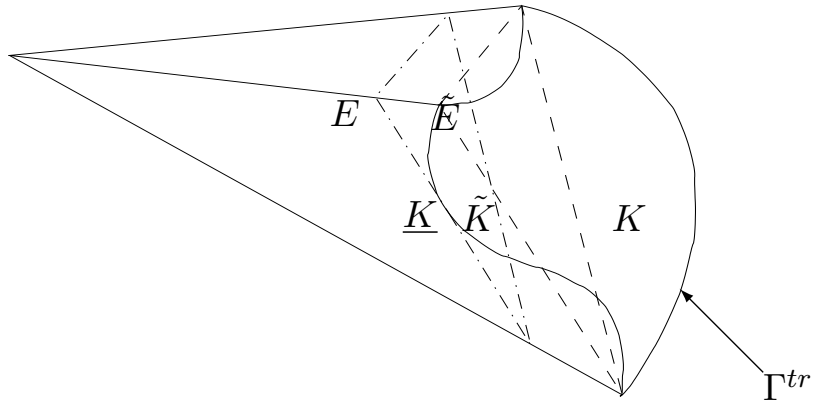

Figure 4: A three-dimensional curved element $K \in \mathcal{T}^{t r}$ (enclosed by the solid lines and curve), its related elements $\tilde{K}$ having the same vertices as $K$ and straight faces (two same faces and the third depicted by a dashed line,) and $\underline{K}$ (having two same faces and the third depicted by a dashed-dotted line.) Although it does not belong to $\Gamma^{t r}$, the face $E$ (enclosed by the solid lines and curve, ) has a curved edge while the related face $\tilde{E}$ (two same faces and the third depicted by a dashed line, ) is a straight triangle.

Assumption $3.1 \mathrm{~b}$ ) is trivially satisfied by shape-regular elements $K$ with straight faces. It is a natural, minimally invasive, condition in view of proving trace estimates, cf. Lemma 4.1 below (see also [1, Theorem 3.10] and [17, Section 3] for illuminating expositions).

Assumption 3.1 a) can always be fulfilled on sufficiently fine meshes, given that the curvature of $\Gamma^{t r}$ is bounded. Moreover, crucially, Assumption 3.1 a) allows for mesh refinement via newest vertex bisection, for it is always possible to subdivide a curved element into two children by drawing a median line/plane segment without crossing the curved element face.

Definition 3.2 For each $K \in \mathcal{T}^{t r}$, we define the simplicial or box-type related element $\tilde{K}$ to be the element with straight/planar faces having the same vertices as $K$. Let also $\underline{K} \subset K$ be the largest sub-element with straight/planar faces and all faces parallel to the faces of the related element $\tilde{K}$.

For two adjacent elements $K, K^{\prime} \in \mathcal{T}^{\text {tr }}$ sharing a common face $E \in \Gamma^{\text {int }} \cup \Gamma^{\text {tr }}$, we shall denote by $\tilde{E}:=\partial \tilde{K} \cap \partial \tilde{K}^{\prime}$ the related common face of the two (also adjacent) related simplicial or prismatic elements $\tilde{K}, \tilde{K}^{\prime}$.

Notice that in general, $K \neq \tilde{K}$ when $\partial K \cap \Gamma^{t r}$ is curved; see Figure 4 for an illustration.

Next, we define

$$
\Gamma_{t r}^{i n t}:=\left\{E \in \Gamma^{i n t}: E \neq \tilde{E}\right\}
$$

i.e., the subset of $\Gamma^{i n t}$ containing all the faces $E \in \Gamma^{i n t}$ with different related faces $\tilde{E}$; see again Figure 4 for an illustration. Notice that $E \neq \tilde{E}$ is possible only when $d=3$.

The above star-shapedness Assumption $3.1 \mathrm{~b}$ ) effectively imply that the angles between the faces $E \subset \Gamma^{t r}$ and those faces in $\Gamma_{t r}^{i n t}$ cannot be arbitrarily small and that the Jacobian of the function parametrising $E \subset \Gamma^{t r}$ on a local coordinate system, as defined above is bounded. Satisfying these assumptions may require a small number of refinements of the elements $K \in \mathcal{T}^{t r}$ of a given initial mesh.

\subsection{Discontinuous Galerkin method}

We define the discontinuous finite element space $S_{h}^{p}$, subortinate to the mesh $\mathcal{T}=\{K\}$, by

$$
S_{h}^{p} \equiv S_{h}^{p}(\mathcal{T})=\left\{v \in L^{2}(\Omega):\left.v\right|_{K} \in \mathcal{P}_{p}(K), K \in \mathcal{T}\right\},
$$


where $\mathcal{P}_{p}(K)$ denotes the space of polynomials of total degree $p$ on an element $K$.

For each element face $E \subset \Gamma^{i n t} \cup \Gamma^{t r}$, there are two elements $K_{1}$ and $K_{2}$ such that $E \subset \partial K_{1} \cap \partial K_{2}$. The outward unit normal vectors on $E$ of $\partial K_{1}$ and $\partial K_{2}$ are denoted by $\mathbf{n}_{K_{1}}$ and $\mathbf{n}_{K_{2}}$, respectively. For a function $v: \Omega \rightarrow \mathbb{R}$ that may be discontinuous across $\Gamma$, we set $v_{i}=\left.v\right|_{K_{i}}$, and we define the jump $\llbracket v \rrbracket$ and the average $\{v\}$ of $v$ across $E$ by

$$
\llbracket v \rrbracket=\left.v\right|_{K_{1}} \mathbf{n}_{K_{1}}+\left.v\right|_{K_{2}} \mathbf{n}_{K_{2}}, \quad\{v\}=\frac{1}{2}\left(\left.v\right|_{K_{1}}+\left.v\right|_{K_{2}}\right) .
$$

Similarly, for a vector valued function $\mathbf{w}$, piecewise smooth on $\mathcal{T}$ with $\mathbf{w}_{i}=\left.\mathbf{w}\right|_{K_{i}}$, we define

$$
\llbracket \mathbf{w} \rrbracket=\left.\mathbf{w}\right|_{K_{1}} \cdot \mathbf{n}_{K_{1}}+\left.\mathbf{w}\right|_{K_{2}} \cdot \mathbf{n}_{K_{2}}, \quad\{\mathbf{w}\}=\frac{1}{2}\left(\left.\mathbf{w}\right|_{K_{1}}+\left.\mathbf{w}\right|_{K_{2}}\right) .
$$

When $E \subset \partial \Omega$, we set $\{v\}=v, \llbracket v \rrbracket=v \mathbf{n}$ and $\llbracket \mathbf{w} \rrbracket=\mathbf{w} \cdot \mathbf{n}$ with $\mathbf{n}$ denoting the outward unit normal to the boundary $\partial \Omega$.

We introduce the meshsize function $\mathbf{h}: \Omega \rightarrow \mathbb{R}$, where $\left.\mathbf{h}\right|_{K}=h_{K}, K \in \mathcal{T}$ and $\mathbf{h}=\{\mathbf{h}\}$ on each $(d-1)$-dimensional open face $E \subset \Gamma$. We also define $h_{\max }:=\max _{x \in \Omega} \mathbf{h}$ and $h_{\min }:=\min _{x \in \Omega} \mathbf{h}$. Without loss of generality, we shall assume that $h_{\max }$ remains uniformly bounded throughout this work, thus, avoiding having estimation constants dependent on $\max \left\{1, h_{\max }\right\}$.

To arrive the interior penalty discontinuous Galerkin method, we multiply (1) by a test function $v \in S_{h}^{p}+\mathcal{H}_{0}^{1}$ and, integrate over each subdomain, we have.

$$
D_{h}\left(u_{h}, v_{h}\right)=\left\langle f, v_{h}\right\rangle, \quad \text { for all } v_{h} \in S_{h}^{p}
$$

where

$$
\begin{aligned}
D_{h}\left(u_{h}, v_{h}\right):= & \sum_{K \in \mathcal{T}} \int_{K} \nabla u_{h} \cdot \nabla v_{h} d x-\int_{\Gamma \backslash \Gamma^{t r}}\left(\left\{\nabla u_{h}\right\} \cdot \llbracket v_{h} \rrbracket+\left\{\nabla v_{h}\right\} \cdot \llbracket u_{h} \rrbracket\right) d s \\
& +\int_{\Gamma \backslash \Gamma^{t r}} \frac{\gamma_{0}}{\mathbf{h}} \llbracket u_{h} \rrbracket \cdot \llbracket v_{h} \rrbracket d s+\int_{\Gamma^{t r}} C_{t r} \llbracket u_{h} \rrbracket \cdot \llbracket v_{h} \rrbracket d s
\end{aligned}
$$

here $\gamma_{0}>0$ is the discontinuity-penalization function (to be defined precisely below,) and $C_{t r}>0$ is the transmission/permeability coefficient. We note that there is no discontinuity penalization on the interface. As is standard in this class of interior penalty dG methods, the penalty parameter has to be chosen large enough in order to ensure the stability of the discontinuous Galerkin discretization; this is the case also here upon excluding the interface terms, cf., [12].

\section{Approximation, trace, and inverse estimates}

We recall some polynomial approximation results over curved elements proven in [13], which are characterized by uniform constants, i.e., constants that are independent on the particular shape of a curved element $K$. Here and in what follows, a generic positive constant, independent of the mesh and problem parameters will be denoted by $C>0$ and may take different values at different instances.

\subsection{Trace and inverse estimates}

Lemma 4.1 Let $v \in H^{1}(K)$ and $K \in \mathcal{T}^{\text {tr }}$. Then, under the above assumptions on the mesh, we have

$$
\|v\|_{\partial K \cap \Gamma^{t r}} \leq C\left(h_{K}^{-1}\|v\|_{K}^{2}+h_{K}\|\nabla v\|_{K}^{2}\right)
$$

with $C>0$, independent of the shape and size of $K$ and of $v$.

Lemma 4.2 Let $K \in \mathcal{T}^{\text {tr }}$ and assume that the related element $\tilde{K}$ is such that

$$
c_{i n v} C_{b} p^{2 d}|K \backslash \tilde{K}|<|K|,
$$


with $c_{i n v}>0$ the constant of the inverse estimate $\|v\|_{L_{\infty}(\tilde{K})}^{2} \leq c_{i n v} p^{2 d}|\tilde{K}|^{-1}\|v\|_{\tilde{K}}^{2}$, for all $v \in \mathcal{P}_{p}(K)$; $C_{b}$ is a geometric constant such that, for each $v \in \mathcal{P}_{p}(K)$, there exists a simplex $K_{b}(v) \subset K$ with straight/planar faces such that $|K| \leq C_{b}\left|K_{b}(v)\right|$ with $\|v\|_{L_{\infty}(K)}=\|v\|_{L_{\infty}\left(K_{b}(v)\right)}$, where the positive constant $C_{b}$ is independent of $v, h_{K}$, and $p$, but depends on the shape-regularity constant of $K$. Then, the following estimate holds

$$
\|v\|_{K}^{2} \leq \theta_{i n v}(K)\|v\|_{K \cap \tilde{K}}^{2}
$$

where $\theta_{\text {inv }}(K):=|K| /\left(|K|-c_{i n v} C_{b} p^{2 d}|K \backslash \tilde{K}|\right)$.

We refer to [13] for the details and the idea behind this non-standard construction.

Lemma 4.3 Let $K \in \mathcal{T}^{\text {tr }}$ and let $\underline{K} \subset K$ and $\tilde{K}$ as in Definition 3.2 be such that

$$
c_{\text {inv }} p^{2 d}|\tilde{K} \backslash \underline{K}|<|\tilde{K}|,
$$

for $c_{i n v}>0$ as in Lemma 4.2. Then, for each $v \in \mathcal{P}_{p}(K)$, the following estimate holds

$$
\|v\|_{\tilde{K}}^{2} \leq \eta_{i n v}(K)\|v\|_{\underline{K}}^{2},
$$

where $\eta_{\text {inv }}(K):=|\tilde{K}| /\left(|\tilde{K}|-c_{\text {inv }} p^{2 d}|\tilde{K} \backslash \underline{K}|\right)$.

Lemma 4.4 Let $K \in \mathcal{T}^{\text {tr }}$ such that a whole face of $K$, say $E_{t r}$, is contained in $\Gamma^{t r}$, and is, in general, curved. Then, for each $v \in \mathcal{P}_{p}(K)$, the inverse estimate

$$
\|v\|_{E_{t r}}^{2} \leq C \frac{p^{2}}{h_{K}}\|v\|_{K}^{2}
$$

with $C>0$ constant, independent of $v, p, h_{K}$ and $K$, but dependent on the shape-regularity constant of $K$.

Lemma 4.5 Let $K \in \mathcal{T}^{\text {tr }}$ and let $E$ a face of $K$, such that $E \subset \partial K \backslash \Gamma^{t r}$. Then, for each $v \in \mathcal{P}_{p}(K)$, the inverse estimate

$$
\|v\|_{E}^{2} \leq C \frac{p^{2}}{h_{K}}\|v\|_{K}^{2},
$$

with $C>0$ constant, independent of $v, p, h_{K}$ and $K$, but dependent on the shape-regularity constant of $K$.

The two constants $\theta_{i n v}$ and $\eta_{i n v}$ from, respectively, Lemma 4.2 and Lemma 4.3 , completely characterise the curvature of the elements in $\mathcal{T}^{t r}$. As such, the following provide an assumption on the mesh curvature necessary for our analysis.

Assumption 4.6 We define the positive function $\theta: L_{2}\left(\Omega_{1} \cup \Omega_{2}\right) \rightarrow \mathbb{R}$ with $\left.\theta\right|_{K}:=\theta_{\text {inv }}(K)$, for $K \in \mathcal{T}^{\text {tr }},\left.\theta\right|_{K}:=1$, for $K \in \mathcal{T} \backslash \mathcal{T}^{\text {tr }}$, and $\theta:=\{\theta\}$ on $\Gamma \backslash \Gamma^{\text {tr }}$. We also define the positive function $\eta: L_{2}\left(\Gamma \backslash \Gamma^{t r}\right) \rightarrow \mathbb{R}$ with $\left.\eta\right|_{E}:=\left\{\eta_{\text {inv }}(E)\right\}$, for $E \in \Gamma \backslash \Gamma^{t r}$. We assume that both functions $\theta$ and $\eta$ are locally quasi-uniform.

\subsection{Recovery operator}

An important tool for the a posteriori analysis will be a conforming recovery operator in the spirit of the original construction by [22]. In [13] we have modified this construction to allow for discontinuous functions across $\Gamma^{t r}$ and for curved elemental faces and edges on $\Gamma^{t r}$. More specifically, the following result was proved.

Lemma 4.7 Given the above mesh assumptions, there exists a recovery operator $\mathcal{E}: S_{h}^{p} \rightarrow \mathcal{H}_{0}^{1}$, such that

$$
\sum_{K \in \mathcal{T}}\left\|\nabla^{\alpha}\left(v_{h}-\mathcal{E}\left(v_{h}\right)\right)\right\|_{K}^{2} \leq C_{\alpha} \sum_{E \subset \Gamma \backslash \Gamma^{t r}}\left\|\sqrt{\theta \eta} \mathbf{h}^{1 / 2-\alpha} \llbracket v_{h} \rrbracket\right\|_{E}^{2},
$$

for all $v_{h} \in S_{h}^{p}, C_{\alpha}>0, \alpha=0,1$, independent of $v_{h}, \theta$ and $\mathbf{h}$.

When $\Gamma^{t r}$ is not curved, i.e., when the mesh $\mathcal{T}$ does not contain any elements with curved faces, we have $\theta=1=\eta$ on $\Gamma \backslash \Gamma^{t r}$ in (10), thereby retrieving the known bound [22, Theorem 2.2]. 


\section{A posteriori error bound}

The dG bilinear form was shown in [13] to be coercive on $D_{h}$ on $S_{h}^{p} \times S_{h}^{p}$, with respect to the dG-norm

$$
\left\|v_{h}\right\| \|:=\left(\sum_{K \in \mathcal{T}}\left\|\nabla v_{h}\right\|_{K}^{2}+\left\|\sqrt{\gamma_{0} / \mathbf{h}} \llbracket v_{h} \rrbracket\right\|_{\Gamma \backslash \Gamma^{t r}}^{2}+C_{t r}\left\|\llbracket v_{h} \rrbracket\right\|_{\Gamma^{t r}}^{2}\right)^{\frac{1}{2}},
$$

for $\gamma_{0} \equiv \gamma_{0}(p)>0$ sufficiently large to be defined precisely below. We note carefully that $\gamma_{0}$ depends only on the polynomial degree $p$ and the shape-regularity of the mesh, through the respective dependence of the inverse estimates from Lemmata 4.4 and 4.5. A posteriori error bounds in the above dG-norm have been proven in [13] and are recalled below.

We begin by considering an inconsistent extension $\hat{D}_{h}:\left(\mathcal{H}_{0}^{1}+S_{h}^{p}\right) \times\left(\mathcal{H}_{0}^{1}+S_{h}^{p}\right) \rightarrow \mathbb{R}$ of $D_{h}$, defined by

$$
\begin{aligned}
\hat{D}_{h}(w, v):= & \sum_{K \in \mathcal{T}} \int_{K} \nabla w \cdot \nabla v d x-\int_{\Gamma \backslash \Gamma^{t r}}(\{\Pi \nabla w\} \cdot \llbracket v \rrbracket+\{\Pi \nabla v\} \cdot \llbracket w \rrbracket) d s \\
& +\int_{\Gamma \backslash \Gamma^{t r}} \frac{\gamma_{0}}{\mathbf{h}} \llbracket w \rrbracket \cdot \llbracket v \rrbracket d s+\int_{\Gamma^{t r}} C_{t r} \llbracket w \rrbracket \cdot \llbracket v \rrbracket d s .
\end{aligned}
$$

Observe that we have $\hat{D}_{h}\left(w_{h}, v_{h}\right)=D_{h}\left(w_{h}, v_{h}\right)$ for all $w_{h}, v_{h} \in S_{h}^{p}$, and $\hat{D}_{h}(w, v)=D(w, v)$ for all $w, v \in \mathcal{H}_{0}^{1}$.

The following continuity and coercivity result holds, whose proof is standard.

Lemma 5.1 For each $w, v \in \mathcal{H}_{0}^{1}+S_{h}^{p}$, we have

$$
\begin{gathered}
\hat{D}_{h}(v, v) \geq \frac{1}{2} \|\left.|v|\right|^{2}, \\
\hat{D}_{h}(w, v) \leq C|\|w\||\||\|v \mid\|,
\end{gathered}
$$

if $\gamma_{0}:=\gamma \theta \eta$ with $\gamma>0$ large enough.

Further we introduce the a posteriori error indicator

$$
\Upsilon:=\left(\sum_{K \in \mathcal{T}} \Upsilon_{K}^{2}\right)^{1 / 2}
$$

with

$$
\Upsilon_{K}:=\left(\Upsilon_{R_{K}}^{2}+\Upsilon_{E_{K}}^{2}+\Upsilon_{J_{K}}^{2}+\Upsilon_{T r_{K}}^{2}\right)^{1 / 2}
$$

comprising of the interior, normal flux, jump and interface residuals

$$
\begin{aligned}
& \Upsilon_{R_{K}}:=\left\|\mathbf{h}\left(\Pi f+\Delta u_{h}\right)\right\|_{K}, \quad \Upsilon_{E_{K}}:=\left\|\sqrt{\mathbf{h}} \llbracket \nabla u_{h} \rrbracket\right\|_{\partial K \cap \Gamma^{i n t}}, \Upsilon_{J_{K}}:=\sqrt{\gamma}\left\|\sqrt{\gamma_{0}} \mathbf{h}^{-1 / 2} \llbracket u_{h} \rrbracket\right\|_{\partial K \cap \Gamma \backslash \Gamma^{t r}}, \\
& \Upsilon_{T_{K}}:=\sum_{i=1}^{2}\left\|\sqrt{\mathbf{h}}\left(C_{t r} \llbracket u_{h} \rrbracket+\nabla u_{h}\right) \cdot \mathbf{n}^{i}\right\|_{\partial K \cap \Gamma^{t r}},
\end{aligned}
$$

where $\Pi: L_{2}(\Omega) \rightarrow S_{h}^{p}$ denotes the orthogonal $L_{2}$-projection operator onto the discontinuous finite element space. We also define the data oscillation term

$$
\Theta_{1}:=\|\mathbf{h}(f-\Pi f)\|
$$

along its restriction on each $K, \Theta_{1, K}:=\|\mathbf{h}(f-\Pi f)\|_{K}$. 
Theorem 5.2 (Upper bound) Let $u$ be the solution of (1) and let $u_{h} \in S_{h}^{p}$ be its dG approximation with $\gamma_{0}$ as in Lemma 5.1. Then, we have the following a posteriori error bound

$$
\left\|u-u_{h}\right\|^{2} \leq C\left(\Upsilon^{2}+\Theta_{1}^{2}\right)+C \sum_{K \in \mathcal{T}}\left(1+\gamma^{-1}\left(1+h_{K} C_{t r}\right)\right) \Upsilon_{J_{K}}^{2}
$$

Theorem 5.3 (Lower bound) Let $u$ be the solution of (1) and let $u_{h} \in S_{h}^{p}$ the $d G$ solution given by (5). Then, for all $K \in \mathcal{T}$, we have the following bound

$$
\Upsilon_{R_{K}}^{2}+\Upsilon_{E_{K}}^{2} \leq C \sum_{K^{\prime} \in \omega_{K}}\left(\theta \eta\left(K^{\prime}\right)\right)^{2}\left(\left\|\nabla\left(u-u_{h}\right)\right\|_{K^{\prime}}^{2}+\Theta_{1, K^{\prime}}^{2}\right)
$$

where $\omega_{K}:=\left\{K^{\prime} \in \mathcal{T}:\right.$ meas $\left._{d-1}\left(\left(\partial K \cap \partial K^{\prime}\right) \backslash \Gamma^{\text {tr }}\right) \neq 0\right\}$. Further, for two elements $K_{i} \in \mathcal{T}^{\text {tr }}$ sharing a face $E \subset \Gamma^{t r}$, we have the bound

$$
\sum_{i=1}^{2}\left\|\sqrt{\mathbf{h}}\left(C_{t r} \llbracket u_{h} \rrbracket+\nabla u_{h}\right) \cdot \tilde{\mathbf{n}}^{i}\right\|_{\tilde{E}_{i}}^{2} \leq C \sum_{i=1}^{2}\left(\left(\theta \eta\left(K_{i}\right)\right)^{2}\left(\left\|\nabla\left(u-u_{h}\right)\right\|_{K_{i}}^{2}+\Theta_{1, K_{i}}^{2}\right)+\Theta_{2, K_{i}}^{2}\right),
$$

where $\tilde{E}_{i}:=\tilde{E} \cap \partial \tilde{K}_{i}, i=1,2$, represent the related faces $\tilde{E}$, signifying that the values of a function on $\tilde{E}_{i}$ are taken from within $\tilde{K}_{i}$. Also, $\tilde{\mathbf{n}}^{i}$ denote the respective outward normal to $\tilde{E}_{i}$. Finally, $\Theta_{2, K_{i}}:=\left|\tilde{K}_{i} \triangle K_{i}\right| h_{K_{i}}^{-d}\left\|C_{t r} \llbracket u_{h} \rrbracket+\nabla u_{h}\right\|_{\tilde{E}_{i}}$ is the interface oscillation term, with $P \triangle Q:=(P \backslash Q) \cup(Q \backslash P)$ denoting the symmetric difference between two sets $P$ and $Q$.

\section{Convergence analysis}

As in the previous section, throughout the analysis we shall assume that the discontinuity-penalisation parameter is given in function of the curvature functions $\theta$ and $\eta$ as $\gamma_{0}:=\gamma \theta \eta$ with $\gamma>0$ large enough in line with Lemma 5.1. We begin by defining

$$
\Upsilon_{\mathcal{T}}^{2}\left(u_{h}\right):=\sum_{K \in \mathcal{T}} h_{K}^{2}\left\|f+\Delta u_{h}\right\|_{K}^{2}+\sum_{E \in \Gamma^{i n t}} h_{E}\left\|\llbracket \nabla u_{h} \rrbracket\right\|_{E}^{2}+\sum_{j=1}^{2}\left\|\mathbf{h}^{1 / 2}\left(C_{t r} \llbracket u_{h} \rrbracket+\nabla u_{h}\right) \cdot \mathbf{n}^{i}\right\|_{\Gamma^{t r}}^{2} .
$$

The following result generalises to the present setting the respective crucial result in [23].

Lemma 6.1 Let $\mathcal{E}\left(u_{h}\right) \in \mathcal{H}_{0}^{1}$ be the continuity recovery of $u_{h}$ given in Lemma 4.7. Then, there exists a constant $\gamma_{\min }>1$, depending only on the shape regularity of the triangulations, such that for each $\gamma \geq \gamma_{\min }$, we have

$$
\gamma\left\|\sqrt{\gamma_{0}} \mathbf{h}^{-1 / 2} \llbracket u_{h} \rrbracket\right\|_{\Gamma \backslash \Gamma^{t r}}^{2} \leq C \Upsilon_{\mathcal{T}}^{2}\left(u_{h}\right),
$$

with $\gamma_{0}=\gamma \theta \eta$.

Proof. The dG method gives

$$
\mathcal{A}:=D_{h}\left(u_{h}-\mathcal{E}\left(u_{h}\right), u_{h}-\mathcal{E}\left(u_{h}\right)\right)=\int_{\Omega} f\left(u_{h}-\mathcal{E}\left(u_{h}\right)\right) d x-D_{h}\left(\mathcal{E}\left(u_{h}\right), u_{h}-\mathcal{E}\left(u_{h}\right)\right) .
$$

Using the continuity of $\mathcal{E}\left(u_{h}\right)$, the above implies

$$
\begin{aligned}
\mathcal{A}= & \int_{\Omega} f\left(u_{h}-\mathcal{E}\left(u_{h}\right)\right) d x-\sum_{K \in \mathcal{T}} \int_{K} \nabla u_{h} \cdot \nabla\left(u_{h}-\mathcal{E}\left(u_{h}\right)\right) d x \\
& +\sum_{K \in \mathcal{T}} \int_{K} \nabla\left(u_{h}-\mathcal{E}\left(u_{h}\right)\right) \cdot \nabla\left(u_{h}-\mathcal{E}\left(u_{h}\right)\right) d x+\int_{\Gamma \backslash \Gamma^{t r}}\left\{\nabla \mathcal{E}\left(u_{h}\right)\right\} \cdot \llbracket u_{h}-\mathcal{E}\left(u_{h}\right) \rrbracket d s \\
& +\int_{\Gamma^{t r}} C_{t r} \llbracket u_{h}-\mathcal{E}\left(u_{h}\right) \rrbracket \cdot \llbracket u_{h}-\mathcal{E}\left(u_{h}\right) \rrbracket d s-\int_{\Gamma^{t r}} C_{t r} \llbracket u_{h} \rrbracket \cdot \llbracket u_{h}-\mathcal{E}\left(u_{h}\right) \rrbracket d s .
\end{aligned}
$$


Integration by parts on the second term on the right-hand side of (21) yields

$$
\begin{aligned}
& -\sum_{K \in \mathcal{T}} \int_{K} \nabla u_{h} \cdot \nabla\left(u_{h}-\mathcal{E}\left(u_{h}\right)\right) d x \\
= & \sum_{K \in \mathcal{T}} \int_{K} \Delta u_{h}\left(u_{h}-\mathcal{E}\left(u_{h}\right)\right) d x-\int_{\Gamma \backslash \Gamma^{t r}}\left\{\nabla u_{h}\right\} \cdot \llbracket u_{h}-\mathcal{E}\left(u_{h}\right) \rrbracket d s-\int_{\Gamma \backslash \Gamma^{t r}}\left\{u_{h}-\mathcal{E}\left(u_{h}\right)\right\} \cdot \llbracket \nabla u_{h} \rrbracket d s \\
& -\sum_{i=1}^{2} \int_{\Gamma^{t r}} n^{i} \cdot \nabla u_{h, i}\left(u_{h, i}-\mathcal{E}\left(u_{h}, i\right)\right) d s,
\end{aligned}
$$

using the notation $v_{i}:=\left.v\right|_{\Omega_{i}}, i=1,2$ for a function $v \in \mathcal{H}_{0}^{1}$. Combining (21) and (22) gives

$$
\begin{aligned}
|\mathcal{A}| \leq & C \Upsilon_{\mathcal{T}}\left(u_{h}\right)\left(\left\|\mathbf{h}^{-1}\left(u_{h}-\mathcal{E}\left(u_{h}\right)\right)\right\|+\left\|\mathbf{h}^{-1 / 2}\left(u_{h}-\mathcal{E}\left(u_{h}\right)\right)\right\|_{\Gamma \backslash \Gamma^{t r}}\right) \\
& +C \Upsilon_{\mathcal{T}}\left(u_{h}\right)\left(\sum_{j=1}^{2}\left\|\mathbf{h}^{-1 / 2}\left(u_{h}-\left.\mathcal{E}\left(u_{h}\right)\right|_{\Omega_{j}}\right)\right\|_{\Gamma_{t r}}^{2}\right)^{1 / 2}+\sum_{K \in \mathcal{T}}\left\|\nabla\left(u_{h}-\mathcal{E}\left(u_{h}\right)\right)\right\|_{K}^{2} \\
& +C_{t r}\left\|\llbracket u_{h}-\mathcal{E}\left(u_{h}\right) \rrbracket\right\|_{\Gamma^{t r}}^{2}+\left\|(\theta \eta / \mathbf{h})^{1 / 2} \llbracket u_{h} \rrbracket\right\|_{\Gamma \backslash \Gamma^{t r}}\left\|(\mathbf{h} / \theta \eta)^{1 / 2}\left\{\nabla \mathcal{E}\left(u_{h}\right)-\nabla u_{h}\right\}\right\|_{\Gamma \backslash \Gamma^{t r}} .
\end{aligned}
$$

Now, applying Lemmas 4.4 and 4.5, along with Lemma 4.7, we arrive at the bound

$$
|\mathcal{A}| \leq C \Upsilon_{\mathcal{T}}\left(u_{h}\right)\left\|\sqrt{\theta \eta} \mathbf{h}^{-1 / 2} \llbracket u_{h} \rrbracket\right\|_{\Gamma \backslash \Gamma^{t r}}+C\left(1+C_{t r} \sqrt{h}_{\max }\right)\left\|\sqrt{\theta \eta} \mathbf{h}^{-1 / 2} \llbracket u_{h} \rrbracket\right\|_{\Gamma \backslash \Gamma^{t r}}^{2} .
$$

Selecting now $\gamma>0$ large enough so that it holds $\frac{\gamma}{4} \geq C\left(1+C_{t r} \sqrt{h}_{\text {max }}\right)$, we have

$$
\begin{aligned}
\left\|\sqrt{\gamma_{0} / \mathbf{h}} \llbracket u_{h} \rrbracket\right\|_{\Gamma \backslash \Gamma^{t r}}^{2} & \leq \frac{C}{\sqrt{\gamma}} \Upsilon_{\mathcal{T}}\left(u_{h}\right)\left\|\sqrt{\gamma_{0}} \mathbf{h}^{-1 / 2} \llbracket u_{h} \rrbracket\right\|_{\Gamma \backslash \Gamma^{t r}}+\frac{1}{4}\left\|\sqrt{\gamma_{0}} \mathbf{h}^{-1 / 2} \llbracket u_{h} \rrbracket\right\|_{\Gamma \backslash \Gamma^{t r}}^{2} \\
& \leq \frac{C}{\gamma} \Upsilon_{\mathcal{T}}^{2}\left(u_{h}\right)+\frac{1}{2}\left\|\sqrt{\gamma_{0}} \mathbf{h}^{-1 / 2} \llbracket u_{h} \rrbracket\right\|_{\Gamma \backslash \Gamma^{t r}}^{2},
\end{aligned}
$$

and, thus, $\gamma\left\|\sqrt{\gamma_{0}} \mathbf{h}^{-1 / 2} \llbracket u_{h} \rrbracket\right\|_{\Gamma \backslash \Gamma^{t r}}^{2} \leq C \Upsilon_{\mathcal{T}}^{2}\left(u_{h}\right)$, which already implies the result.

Therefore, under the assumptions of Lemma 6.1, the a posteriori bound from Theorem 5.2 can be reduced to

$$
\left\|u-u_{h}\right\|^{2} \leq C \Upsilon_{\mathcal{T}}^{2}\left(u_{h}\right),
$$

i.e., the penalty term disappears from the estimator. This is crucial in view of proving the estimator reduction property, since the penalty term involves the mesh-size $h$ with a negative power.

\subsection{Adaptive procedure}

We consider a sequence $\left\{S_{m}\right\}_{m \in \mathbb{N}_{0}}$ of fitted dG spaces $S_{m} \equiv S_{h_{m}}^{p}$ subordinate to a mesh $\mathcal{T}_{m}$, constructed satisfying the above assumptions. We shall describe and analyze an adaptive discontinuous Galerkin method defined by an iteration of the form SOLVE $\rightarrow$ ESTIMATE $\rightarrow$ MARK $\rightarrow$ REFINE, which will determine $\left\{S_{m}\right\}_{m \in \mathbb{N}}$ automatically.

SOLVE: Given a mesh $\mathcal{T}_{m}$, the $\mathrm{dG}$ approximation $u_{m} \in S_{m}$ is computed by solving

$$
D_{m}\left(u_{m}, v_{m}\right)=\left\langle f, v_{m}\right\rangle \quad \forall v_{m} \in S_{m}
$$

with $D_{m}$ denoting the discrete bilinear form $D_{h}$ from (6) with respect to the mesh $\mathcal{T}_{m}$.

ESTIMATE: For each element $K \in \mathcal{T}_{m}$, we evaluate the local a posteriori error estimators $\Upsilon_{\mathcal{T}_{m}}\left(u_{m}, K\right)$, given by

$$
\begin{aligned}
\Upsilon_{\mathcal{T}_{m}}^{2}\left(u_{m}, K\right):= & \sum_{K \in \mathcal{T}_{m}} h_{K}^{2}\left\|f+\Delta u_{m}\right\|_{K}^{2}+\sum_{E \in \partial K \cap \Gamma_{m}^{i n t}} h_{E}\left\|\llbracket \nabla u_{m} \rrbracket\right\|_{E}^{2} \\
& +\sum_{j=1}^{2}\left\|\mathbf{h}^{1 / 2}\left(C_{t r} \llbracket u_{m} \rrbracket+\nabla u_{m}\right) \cdot \mathbf{n}^{i}\right\|_{\partial K \cap \Gamma_{m}^{t r}}^{2}
\end{aligned}
$$


note that, with this notation, we have

$$
\Upsilon_{\mathcal{T}_{m}}^{2}\left(u_{m}\right)=\sum_{K \in \mathcal{T}_{m}} \Upsilon_{\mathcal{T}_{m}}^{2}\left(u_{m}, K\right)
$$

MARK: The third step is based on the, so-called, Dörfler or bulk marking strategy [16], whereby, given $0<\mu<1$, we find a collection of elements $\mathcal{M}_{m} \subset \mathcal{T}_{m}$ such that

$$
\mu \Upsilon_{\mathcal{T}_{m}}^{2}\left(u_{m}\right) \leq \Upsilon_{\mathcal{T}_{m}}^{2}\left(u_{m}, \mathcal{M}_{m}\right):=\sum_{K \in \mathcal{M}_{m}} \Upsilon_{\mathcal{T}_{m}}^{2}\left(u_{m}, K\right)
$$

the collection $\mathcal{M}_{m}$ is called the set of marked elements.

REFINE: Finally, the elements and faces that have been marked are subdivided by bisection into children or, more in general, by at least $r \geq 1$ bisections, see below (cf., [16]). This process determines the new mesh $\mathcal{T}_{m}$. A crucial challenge for the interface problem compared to the, now standard, proof of convergence of the above adaptive procedure applied to the single domain problem, is that the steps MARK and REFINE are required to retain the mesh assumptions above for the elements admitting curved faces.

In what follows, we shall use a subscript or superscript $m$ to denote quantities related to mesh $\mathcal{T}_{m}$. For instance, the penalisation parameter associated to $\mathcal{T}_{m}$ will be denoted by $\gamma_{0}^{m}=\gamma \theta_{m} \eta_{m}$, cf. Sections 6 and 5. Note indeed that the penalisation parameter depends on the mesh through the functions $\theta \eta$ measuring how far a given element is from being straight. Instead, we assume that $\gamma>1$ is fixed for all elements and meshes. Similarly $\|\cdot \cdot\|_{m}$ denotes the dG-norm corresponding to $\mathcal{T}_{m}$. We shall omit the index when no confusion is likely to occur. For the convergence proof, we make the following (mild) assumption.

Assumption 6.2 Let $\theta_{m}, \eta_{m}$ denote the parameters characterising the curvature of mesh from Assumption 4.6. We further require

$$
1 \leq \theta_{m} \eta_{m} \leq C_{\gamma}
$$

with $C_{\gamma}>1$ a constant independent of $m \in \mathbb{N}_{0}$.

Assumption 6.2 immediately implies the bounds:

$$
\gamma_{0}^{m} \leq \gamma C_{\gamma} \quad \text { and } \quad \max \left\{\frac{\gamma_{0}^{m}}{\gamma_{0}^{m-1}}, \frac{\gamma_{0}^{m-1}}{\gamma_{0}^{m}}\right\} \leq C_{\gamma}
$$

where $\gamma_{0}^{n}=\gamma \theta_{n} \eta_{n}, n \in \mathbb{N}_{0}$.

Of course, Assumption 6.2 can always be satisfied upon judicious choice of the penalty parameter $\gamma$ and, if needed, after a finite number of uniform refinements of the mesh in the vicinity of the interface.

Now, let $\beta_{1}$ and $\beta_{2}$ be two constants depending only on the initial triangulation and such that $0<\beta_{1}<\beta_{2}<1$.

Assumption 6.3 For each element refined at the $(m-1)$-st iteration, i.e. for each $K \in \mathcal{M}_{m-1}$, let $\mathcal{R}_{K}:=\left\{K^{\prime} \in \mathcal{T}_{m}: K^{\prime} \subset K\right\}$ be the corresponding patch of elements in the new mesh. It is assumed that all $\mathcal{R}_{K}$ have been obtained from $K \in \mathcal{M}_{m-1}$ by at least $r$ refinements and

$$
\kappa_{1} h_{K^{\prime}} \leq h_{K} \leq \kappa_{2} h_{K^{\prime}}
$$

where $\kappa_{1}:=2^{r / d} \beta_{1} / \beta_{2}$ and $\kappa_{2}:=2^{r / d} \beta_{2} / \beta_{1}$.

For elements $T \in \mathcal{T} \backslash \mathcal{T}^{t r}$, Assumption 6.3 is known to be satisfied when $\mathcal{T}_{m}$ is constructed from $\mathcal{T}_{m-1}$ via a newest vertex bisection technique, see, e.g., [14]; for elements with faces on $\Gamma^{t r}$, Assumption 6.3 can always be satisfied after a finite number of suitable uniform refinements in the vicinity of the interface, if needed.

Next, we show an estimator reduction property (cf., [15], Corollary 4.4). 
Lemma 6.4 Let $\mu$ and $r$ be, respectively, the parameters determining the bulk marking and the number of bisections in the adaptive algorithm of Section 6.1. Assuming that $\delta:=1-\frac{\beta_{2}}{\beta_{1}} 2^{-r / d}<1$ and $0<\mu<1$, we have

$$
\begin{aligned}
\Upsilon_{\mathcal{T}_{m}}^{2}\left(u_{m}\right) \leq & \left(1-\frac{\delta \mu}{2}\right) \Upsilon_{\mathcal{T}_{m-1}}^{2}\left(u_{m-1}\right)+\left(1+\frac{2}{\delta \mu}\right) \max \left\{1, C_{t r}\right\} \\
& \times\left(\sum_{K \in \mathcal{T}_{m}}\left\|\nabla\left(u_{m}-u_{m-1}\right)\right\|_{K}^{2}+\left\|\sqrt{\theta_{m} \eta_{m} / \mathbf{h}_{m}} \llbracket u_{m}-u_{m-1} \rrbracket\right\|_{\Gamma_{m} \backslash \Gamma_{m}^{t r}}^{2}\right) .
\end{aligned}
$$

Proof. In what follows mesh dependent quantities without index values refer to $\mathcal{T}_{m}$. Set $v_{m}:=$ $u_{m}-u_{m-1}, m \in \mathbb{N}$, for brevity. Using a standard Poincaré-Friedrichs inequality along with Lemma 4.7, we have

$$
\begin{aligned}
\left\|v_{m}\right\|^{2} & \leq 2\left\|\mathcal{E}\left(v_{m}\right)\right\|^{2}+2\left\|v_{m}-\mathcal{E}\left(v_{m}\right)\right\|^{2} \\
& \leq C \sum_{j=1,2}\left\|\nabla \mathcal{E}\left(v_{m}\right)\right\|_{\Omega_{j}}^{2}+C\left\|\sqrt{\theta_{m} \eta_{m} \mathbf{h}_{m}} \llbracket v_{m} \rrbracket\right\|_{\Gamma_{m} \backslash \Gamma_{m}^{t r}}^{2} \\
& \leq C \sum_{K \in \mathcal{T}_{m}}\left(\left\|\nabla v_{m}\right\|_{K}^{2}+\left\|\nabla\left(v_{m}-\mathcal{E}\left(v_{m}\right)\right)\right\|_{K}^{2}\right)+C\left\|\sqrt{\theta_{m} \eta_{m} \mathbf{h}_{m}} \llbracket v_{m} \rrbracket\right\|_{\Gamma_{m} \backslash \Gamma_{m}^{t r}}^{2} \\
& \leq C \sum_{K \in \mathcal{T}_{m}}\left\|\nabla v_{m}\right\|_{K}^{2}+C\left\|\sqrt{\theta_{m} \eta_{m} / \mathbf{h}_{m}} \llbracket v_{m} \rrbracket\right\|_{\Gamma_{m} \backslash \Gamma_{m}^{t r}}^{2} .
\end{aligned}
$$

Therefore, using Lemma 4.4 and (32), we have

$$
\begin{aligned}
& \sum_{K \in \mathcal{T}_{m}}\left\|\mathbf{h}_{m} \Delta v_{m}\right\|_{K}^{2}+\left\|\mathbf{h}_{m}^{1 / 2} \llbracket \nabla v_{m} \rrbracket\right\|_{\Gamma_{m}^{i n t}}^{2}+\sum_{j=1,2} C_{t r}\left\|\mathbf{h}_{m}^{1 / 2}\left(\llbracket v_{m} \rrbracket+\nabla v_{m}\right) \cdot \mathbf{n}^{i}\right\|_{\Gamma_{m}^{t r}}^{2} \\
\leq & C \sum_{K \in \mathcal{T}_{m}}\left\|\nabla v_{m}\right\|_{K}^{2}+C C_{t r}\left\|v_{m}\right\|^{2} \leq C \sum_{K \in \mathcal{T}_{m}}\left\|\nabla v_{m}\right\|_{K}^{2}+C C_{t r}\left\|\sqrt{\theta_{m} \eta_{m} / \mathbf{h}_{m}} \llbracket v_{m} \rrbracket\right\|_{\Gamma_{m} \backslash \Gamma_{m}^{t r}}^{2},
\end{aligned}
$$

for $C>0$ constant depending only on the local geometry of the triangulation.

Using the elementary identity $(a+b)^{2} \leq(1+\lambda) a^{2}+\left(1+\lambda^{-1}\right) b^{2}$, for $a, b, \lambda \in \mathbb{R}, \lambda>0$, we have

$$
\begin{gathered}
\sum_{K \in \mathcal{T}_{m}}\left\|\mathbf{h}_{m}\left(f+\Delta u_{m}\right)\right\|_{K}^{2} \leq(1+\lambda) \sum_{K \in \mathcal{T}_{m}}\left\|\mathbf{h}_{m}\left(f+\Delta u_{m-1}\right)\right\|_{K}^{2}+\left(1+\lambda^{-1}\right) \sum_{K \in \mathcal{T}_{m}}\left\|\mathbf{h}_{m} \Delta v_{m}\right\|_{K}^{2}, \\
\left\|\mathbf{h}_{m}^{1 / 2} \llbracket \nabla u_{m} \rrbracket\right\|_{\Gamma_{m}^{i n t}}^{2} \leq(1+\lambda)\left\|\mathbf{h}_{m}^{1 / 2} \llbracket \nabla u_{m-1} \rrbracket\right\|_{\Gamma_{m}^{i n t}}^{2}+\left(1+\lambda^{-1}\right)\left\|\mathbf{h}_{m}^{1 / 2} \llbracket \nabla v_{m} \rrbracket\right\|_{\Gamma_{m}^{i n t}}^{2},
\end{gathered}
$$

and

$$
\begin{aligned}
\sum_{j=1,2}\left\|\mathbf{h}_{m}^{1 / 2}\left(\llbracket u_{m} \rrbracket+\nabla u_{m}\right) \cdot \mathbf{n}^{i}\right\|_{\Gamma_{m}^{t r}}^{2} \leq & (1+\lambda) \sum_{j=1,2}\left\|\mathbf{h}_{m}^{1 / 2}\left(\llbracket u_{m-1} \rrbracket+\nabla u_{m-1}\right) \cdot \mathbf{n}^{i}\right\|_{\Gamma_{m}^{t r}}^{2} \\
& +\left(1+\lambda^{-1}\right) \sum_{j=1,2}\left\|\mathbf{h}_{m}^{1 / 2}\left(\llbracket v_{m} \rrbracket+\nabla v_{m}\right) \cdot \mathbf{n}^{i}\right\|_{\Gamma_{m}^{t r}}^{2} .
\end{aligned}
$$

Combining (34), (35) and (36), with (33), we deduce

$$
\Upsilon_{\mathcal{T}_{m}}^{2}\left(u_{m}\right) \leq(1+\lambda) \Upsilon_{\mathcal{T}_{m}}^{2}\left(u_{m-1}\right)+\left(1+\lambda^{-1}\right) C\left(\sum_{K \in \mathcal{T}_{m}}\left\|\nabla v_{m}\right\|_{K}^{2}+\left\|\sqrt{\theta_{m} \eta_{m} / \mathbf{h}_{m}} \llbracket v_{m} \rrbracket\right\|_{\Gamma_{m} \backslash \Gamma_{m}^{t_{m}}}^{2}\right) .
$$

From Assumption 6.3, for all $K^{\prime} \in \mathcal{R}_{K}$, we have $h_{K^{\prime}} \leq\left(\frac{\beta_{2}}{\beta_{1}}\right) 2^{-r / d} h_{K}$ and

$$
\begin{aligned}
\Upsilon_{\mathcal{T}_{m}}^{2}\left(u_{m-1}\right)= & \Upsilon_{\mathcal{T}_{m-1}}^{2}\left(u_{m-1}, \mathcal{T}_{m-1} \backslash \mathcal{M}_{m-1}\right)+\Upsilon_{\mathcal{T}_{m}}^{2}\left(u_{m-1},\left\{\mathcal{R}_{K}: K \in \mathcal{M}_{m-1}\right\}\right) \\
& \leq \Upsilon_{\mathcal{T}_{m-1}}^{2}\left(u_{m-1}, \mathcal{T}_{m-1} \backslash \mathcal{M}_{m-1}\right)+2^{-1 / 2} \Upsilon_{\mathcal{T}_{m-1}}^{2}\left(u_{m-1}, \mathcal{M}_{m-1}\right) \\
& \leq \Upsilon_{\mathcal{T}_{m-1}}^{2}\left(u_{m-1}, \mathcal{T}_{m-1}\right)-\left(1-\frac{\beta_{2}}{\beta_{1}} 2^{-r / d}\right) \Upsilon_{\mathcal{T}_{m-1}}^{2}\left(u_{m-1}, \mathcal{M}_{m-1}\right) \\
& \leq(1-\delta \mu) \Upsilon_{\mathcal{T}_{m-1}}^{2}\left(u_{m-1}, \mathcal{T}_{m-1}\right)
\end{aligned}
$$

making use of the Dörfler-type marking strategy property (28) in the last step. Substituting (38) into (37), and selecting $\lambda=\delta \mu / 2$, the result already follows by noting that $\left(1+\frac{\delta \mu}{2}\right)(1-\delta \mu) \leq 1-\frac{\delta \mu}{2}$. 


\subsection{Quasi-orthogonality}

We now prove a mesh perturbation result, which we shall use to establish the quasi-orthogonality in Lemma 6.6 below.

Lemma 6.5 For $\gamma>1$ sufficiently large, we have

$$
\hat{D}_{m}(z, z) \leq(1+\epsilon) \hat{D}_{m-1}(z, z) x+\frac{\xi \gamma}{\epsilon}\left\|\sqrt{\gamma_{0}^{m-1} / \mathbf{h}_{m-1}} \llbracket z \rrbracket\right\|_{\Gamma_{m-1} \backslash \Gamma_{m-1}^{t r}}^{2},
$$

for all $z \in S_{m-1}+\mathcal{H}_{0}^{1}$, and for any $0<\epsilon<1$, and $\xi:=C_{\gamma} 2^{(r+1) / d} \beta_{2} / \beta_{1}$, with $r \in \mathbb{N}$ denoting the minimum number of refinements of the elements on $\Gamma^{\text {tr }}$ required in the transition from $\mathcal{T}_{m-1}$ to $\mathcal{T}_{m}$; here, $\hat{D}_{m} \equiv \hat{D}_{h}^{m}$.

Proof. Since $\mathcal{T}_{m}$ is a refinement of $\mathcal{T}_{m-1}$, we have $z \in S_{m}+\mathcal{H}_{0}^{1}$ also. Hence,

$$
\begin{aligned}
& \hat{D}_{m}(z, z)=\hat{D}_{m-1}(z, z)+2 \int_{\Gamma_{m} \backslash \Gamma_{m}^{t r}}\{\Pi \nabla z\} \cdot \llbracket z \rrbracket d s-2 \int_{\Gamma_{m-1} \backslash \Gamma_{m-1}^{t r}}\{\Pi \nabla z\} \cdot \llbracket z \rrbracket d s \\
& +\left\|\sqrt{\gamma_{0}^{m} / \mathbf{h}_{m}} \llbracket z \rrbracket\right\|_{\Gamma_{m} \backslash \Gamma_{m}^{t r}}^{2}-\left\|\sqrt{\gamma_{0}^{m-1} / \mathbf{h}_{m-1}} \llbracket z \rrbracket\right\|_{\Gamma_{m-1} \backslash \Gamma_{m-1}^{t r}}^{2},
\end{aligned}
$$

since

$$
\|\llbracket z \rrbracket\|_{\Gamma_{m}^{t r}}^{2}=\|\llbracket z \rrbracket\|_{\Gamma_{m-1}^{t r}}^{2}, \quad \sum_{K \in \mathcal{T}_{m}}\|\nabla z\|^{2}=\sum_{K \in \mathcal{T}_{m-1}}\|\nabla z\|^{2}
$$

Using standard arguments based on inverse inequalities gives

$$
\begin{aligned}
& 2 \int_{\Gamma_{m-1} \backslash \Gamma_{m-1}^{t r}}\{\Pi \nabla z\} \cdot \llbracket z \rrbracket d s \leq C\left(\sum_{K \in \mathcal{T}_{m-1}}\left\|\sqrt{h_{m-1} / \gamma \gamma_{0}^{m-1}} \nabla z\right\|_{K}^{2}\right)^{1 / 2}\left\|\sqrt{\gamma \gamma_{0}^{m-1} / h_{m-1}} \llbracket z \rrbracket\right\|_{\Gamma_{m-1} \backslash \Gamma_{m-1}^{t r}} \\
& \leq \frac{\epsilon}{2} \hat{D}_{m-1}(z, z)+\frac{\gamma}{2 \epsilon}\left\|\sqrt{\gamma_{0}^{m-1} / \mathbf{h}_{m-1}} \llbracket z \rrbracket\right\|_{\Gamma_{m-1} \backslash \Gamma_{m-1}^{t r}}^{2},
\end{aligned}
$$

and, similarly,

$$
\begin{aligned}
2 \int_{\Gamma_{m} \backslash \Gamma_{m}^{t r}}\{\Pi \nabla z\} \cdot \llbracket z \rrbracket d s & \leq C\left(\sum_{K \in \mathcal{T}_{m}}\left\|\sqrt{h_{m} / \gamma \gamma_{0}^{m}} \nabla z\right\|_{K}^{2}\right)^{1 / 2}\left\|\sqrt{\gamma \gamma_{0}^{m} / h_{m}} \llbracket z \rrbracket\right\|_{\Gamma_{m} \backslash \Gamma_{m}^{t r}} \\
& \leq \frac{\epsilon}{2} \hat{D}_{m-1}(z, z)+\frac{\gamma}{2 \epsilon}\left\|\sqrt{\gamma_{0}^{m} / \mathbf{h}_{m}} \llbracket z \rrbracket\right\|_{\Gamma_{m} \backslash \Gamma_{m}^{t r}}^{2},
\end{aligned}
$$

for $\gamma>1$ sufficiently large. Using (30) and Assumption 6.3, we also have

$$
\left\|\sqrt{\gamma_{0}^{m}} \mathbf{h}_{\mathbf{m}}{ }^{-1 / 2} \llbracket z \rrbracket\right\|_{\Gamma_{m} \backslash \Gamma_{m}^{t r}}^{2} \leq C_{\gamma} 2^{r / d} \beta_{2} / \beta_{1}\left\|\sqrt{\gamma_{0}^{m-1}} \mathbf{h}_{\mathbf{m}-\mathbf{1}}{ }^{-1 / 2} \llbracket z \rrbracket\right\|_{\Gamma_{m-1} \backslash \Gamma_{m-1}^{t r}}^{2} .
$$

Combining the above bounds yields

$$
\hat{D}_{m}(z, z) \leq(1+\epsilon) \hat{D}_{m-1}(z, z)+C\left\|\sqrt{\gamma_{0}^{m-1} / \mathbf{h}_{m-1}} \llbracket z \rrbracket\right\|_{\Gamma_{m-1} \backslash \Gamma_{m-1}^{t r}}^{2},
$$

with $C=\left(\frac{\gamma}{2 \epsilon}-1\right)+\left(1+\frac{\gamma}{2 \epsilon}\right) C_{\gamma} 2^{r / d} \beta_{2} / \beta_{1}$, and the assertion follows by noting that $C_{\gamma} 2^{r / d} \beta_{2} / \beta_{1}>1$.

Lemma 6.6 Let $e_{m}:=u-u_{m}, \quad e_{m-1}:=u-u_{m-1}$ and $0<\epsilon<1$. Then we have

$$
\hat{D}_{m}\left(e_{m}, e_{m}\right)=(1+\epsilon) \hat{D}_{m-1}\left(e_{m-1}, e_{m-1}\right)+\frac{C}{\gamma}\left(\epsilon^{-1} \Upsilon_{\mathcal{T}_{m-1}}^{2}\left(u_{m-1}\right)+\Upsilon_{\mathcal{T}_{m}}^{2}\left(u_{m}\right)\right)-\frac{1}{4}\left\|u_{m}-u_{m-1}\right\|_{m}^{2},
$$

for $\gamma>1$ sufficiently large and for some $C>0$ constant, independent of the functions and the parameters written explicitly in the bound. 
Proof. For brevity, we set $u_{m}^{d}:=u_{m}-\mathcal{E}\left(u_{m}\right), m \in \mathbb{N}$. Noting that $\mathcal{E}\left(u_{m}\right)-\mathcal{E}\left(u_{m-1}\right) \in S_{m} \cap \mathcal{H}_{0}^{1}$, Galerkin orthogonality and the symmetry of the bilinear form, along with the identity

$$
e_{m}+\mathcal{E}\left(u_{m}\right)-\mathcal{E}\left(u_{m-1}\right)=e_{m-1}+u_{m-1}^{d}-u_{m}^{d},
$$

imply

$$
\begin{aligned}
\hat{D}_{m}\left(e_{m}, e_{m}\right)= & \hat{D}_{m}\left(e_{m}+\mathcal{E}\left(u_{m}\right)-\mathcal{E}\left(u_{m-1}\right), e_{m}+\mathcal{E}\left(u_{m}\right)-\mathcal{E}\left(u_{m-1}\right)\right) \\
& -\hat{D}_{m}\left(\mathcal{E}\left(u_{m}\right)-\mathcal{E}\left(u_{m-1}\right), \mathcal{E}\left(u_{m}\right)-\mathcal{E}\left(u_{m-1}\right)\right) \\
= & \hat{D}_{m}\left(e_{m-1}, e_{m-1}\right)+2 \hat{D}_{m}\left(e_{m-1}, u_{m-1}^{d}-u_{m}^{d}\right) \\
& +\hat{D}_{m}\left(u_{m-1}^{d}-u_{m}^{d}, u_{m-1}^{d}-u_{m}^{d}\right)-\hat{D}_{m}\left(\mathcal{E}\left(u_{m}\right)-\mathcal{E}\left(u_{m-1}\right), \mathcal{E}\left(u_{m}\right)-\mathcal{E}\left(u_{m-1}\right)\right) .
\end{aligned}
$$

The last term on the right hand side of (43) can be bounded as follows

$$
\begin{aligned}
& \hat{D}_{m}\left(\mathcal{E}\left(u_{m}\right)-\mathcal{E}\left(u_{m-1}\right), \mathcal{E}\left(u_{m}\right)-\mathcal{E}\left(u_{m-1}\right)\right) \\
\geq & \frac{1}{2}\left|\left\|u_{m-1}-u_{m}\right\|_{m}^{2}+\frac{1}{2}\right| \mid \mathcal{E}\left(u_{m}\right)-u_{m}+u_{m-1}-\mathcal{E}\left(u_{m-1}\right) \|_{m}^{2} \\
& -\left|\left\langle u_{m}-u_{m-1}, \mathcal{E}\left(u_{m}\right)-u_{m}+u_{m-1}-\mathcal{E}\left(u_{m-1}\right)\right\rangle\right| \\
& \geq \frac{1}{4}\left|\left\|u_{m}-u_{m-1}\right\|_{m}^{2}-\frac{1}{2}\right|\left\|u_{m-1}^{d}-u_{m}^{d}\right\|_{m}^{2} .
\end{aligned}
$$

Using this bound and Lemma 6.5 in (43), together with the continuity and the coercivity of $\hat{D}_{m}$, gives

$$
\begin{aligned}
\hat{D}_{m}\left(e_{m}, e_{m}\right) \leq & (1+\epsilon) \hat{D}_{m-1}\left(e_{m-1}, e_{m-1}\right)+\frac{\xi \gamma}{\epsilon}\left\|\sqrt{\gamma_{0}^{m-1} / \mathbf{h}_{m-1}} \llbracket e_{m-1}\right\| \|_{\Gamma_{m-1} \backslash \Gamma_{m-1}^{t r}}^{2}, \\
& +C\left\|u_{m-1}^{d}-u_{m}^{d}\right\|_{m}^{2}-\frac{1}{4}\left\|u_{m}-u_{m-1}\right\| \|_{m}^{2},
\end{aligned}
$$

for some $\epsilon>0$, with $C>1$ a constant only depending on the continuity constant in Lemma 5.1.

Finally, using the triangle inequality, in conjunction with Lemma (4.7), under the assumptions of Lemma 6.1, along wth (30) and Assumption 6.3, we have

$$
\begin{aligned}
\left\|u_{m-1}^{d}-u_{m}^{d}\right\|_{m}^{2} & \leq C C_{\gamma} 2^{r / d} \gamma\left\|\sqrt{\gamma_{0}^{m-1}} \mathbf{h}_{\mathbf{m}-\mathbf{1}}{ }^{-1 / 2} \llbracket u_{m-1} \rrbracket\right\|_{\Gamma_{m-1} \backslash \Gamma_{m-1}^{t r}}^{2}+C \gamma\left\|\sqrt{\gamma_{0}^{m}} \mathbf{h}_{\mathbf{m}}{ }^{-1 / 2} \llbracket u_{m} \rrbracket\right\|_{\Gamma_{m} \backslash \Gamma_{m}^{t r}}^{2} \\
& \leq \frac{C}{\gamma}\left(\Upsilon_{\mathcal{T}_{m-1}}^{2}\left(u_{m-1}\right)+\Upsilon_{\mathcal{T}_{m}}^{2}\left(u_{m}\right)\right),
\end{aligned}
$$

and the result readily follows.

\subsection{Contraction property}

We are now ready to prove the main theorem of this work.

Theorem 6.7 Let $u \in \mathcal{H}_{0}^{1}$. Then, there exist constants $\beta>0$ and $0<\rho<1$ depending only on the shape regularity of the triangulations and on the marking parameter $\mu$, such that

$$
\hat{D}_{m}\left(e_{m}, e_{m}\right)+\beta \Upsilon_{\mathcal{T}_{m}}^{2}\left(u_{m}\right) \leq \rho\left(\hat{D}_{m-1}\left(e_{m-1}, e_{m-1}\right)+\beta \Upsilon_{\mathcal{T}_{m-1}}^{2}\left(u_{m-1}\right)\right) .
$$

Proof. Using Lemmas 6.6 and 6.4, we have

$$
\begin{aligned}
& \hat{D}_{m}\left(e_{m}, e_{m}\right)+\beta \Upsilon_{\mathcal{T}_{m}}^{2}\left(u_{m}\right) \\
\leq & (1+\epsilon) \hat{D}_{m-1}\left(e_{m-1}, e_{m-1}\right)-\frac{1}{4}\left\|u_{m}-u_{m-1}\right\|_{m}^{2}+\frac{C}{\epsilon \gamma} \Upsilon_{\mathcal{T}_{m-1}}^{2}\left(u_{m-1}\right)+\left(\frac{C}{\gamma}+\beta\right) \Upsilon_{\mathcal{T}_{m}}^{2}\left(u_{m}\right) \\
\leq & (1+\epsilon) \hat{D}_{m-1}\left(e_{m-1}, e_{m-1}\right)-\frac{1}{4}\left\|u_{m}-u_{m-1}\right\|_{m}^{2}+\left(\frac{C}{\epsilon \gamma}+\left(\frac{C}{\gamma}+\beta\right)\left(1-\frac{\delta \mu}{2}\right)\right) \Upsilon_{\mathcal{T}_{m-1}}^{2}\left(u_{m-1}\right) \\
& +\left(1+\frac{2}{\delta \mu}\right)\left(\frac{C}{\gamma}+\beta\right) \max \left\{1, C_{t r}\right\}\left(\sum_{K \in \mathcal{T}_{m}}\left\|\nabla\left(u_{m}-u_{m-1}\right)\right\|_{K}^{2}+\left\|\sqrt{\theta_{m} \eta_{m} / \mathbf{h}_{m}} \llbracket u_{m}-u_{m-1} \rrbracket\right\|_{\Gamma_{m} \backslash \Gamma_{m}^{t r}}^{2}\right),
\end{aligned}
$$


for any $\epsilon>0$. If we choose $\beta=1 /\left(4\left(1+\frac{2}{\delta \mu}\right) \max \left\{1, C_{t r}\right\}\right)-C / \gamma$, and $\gamma$ large enough (to be defined precisely below), this leads to absorption of the fourth term on the right hand side of (45) by $\left\|\left|u_{m-1}-u_{m}\right|\right\|_{m}$. Also, from (25), we have $\epsilon \hat{D}_{m-1}\left(e_{m-1}, e_{m-1}\right) \leq \epsilon \Upsilon_{\mathcal{T}_{m-1}}^{2}\left(u_{m-1}\right)$. Therefore, for $\epsilon=4 C /(\gamma \beta \delta \mu)$ and $\gamma$ large enough, we deduce

$$
\hat{D}_{m}\left(e_{m}, e_{m}\right)+\beta \Upsilon_{\mathcal{T}_{m}}^{2}\left(u_{m}\right) \leq \hat{D}_{m-1}\left(e_{m-1}, e_{m-1}\right)+\left(\beta\left(1-\frac{\delta \mu}{4}\right)+\frac{C}{\gamma}\left(1-\frac{\delta \mu}{2}\right)\right) \Upsilon_{\mathcal{T}_{m-1}}^{2}\left(u_{m-1}\right) .
$$

and the proof already follows by choosing $\gamma \geq 4 C(1-\delta \mu / 2) /(\beta \delta \mu)$ so that $\beta>0$.

The contraction of $\hat{D}_{m}\left(e_{m}, e_{m}\right)+\beta \Upsilon_{\mathcal{T}_{m}}^{2}\left(u_{m}\right)$ as $m \rightarrow \infty$ implies that $\hat{D}_{m}\left(e_{m}, e_{m}\right) \rightarrow 0$ as $m \rightarrow \infty$, which, in turn, implies $\left\|e_{m}\right\| \| \rightarrow 0$ as $m \rightarrow \infty$. Therefore the above adaptive algorithm converges.

\section{Numerical experiments}

We shall now illustrate the practical performance of the adaptive algorithm analysed above. All results shown are obtained with an implementation based on the deal. II finite element library [2]. Here, we present the result of two numerical examples, with $\gamma=10$ and local polynomial degrees equal to one and two. In all the cases, the starting mesh is the uniform square mesh with $16 \times 16$ elements. Although not discussed above merely for simplicity of the presentation, it is straightforward to extend the proposed $\mathrm{dG}$ method to problems with non-homogeneous Dirichlet boundary conditions on the external boundary. Moreover, the examples presented below are concerned with straight interfaces; for an example with curved interface we refer to [13].

\subsection{Example 1}

Let $\Omega=(-1,1)^{2}$ and $\Omega_{1}=(-1,0) \times(-1,1), \Omega_{2}=(0,1) \times(-1,1)$, i.e., the interval $\Gamma^{t r}=\{(x, y)$ : $x=0,0<y<1\}$. The Dirichlet boundary conditions and source term $f$ are determined by the exact solution

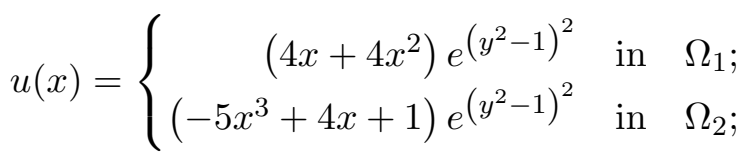

both components are subject to non-homogeneous Dirichlet boundary condition. The solution $u$ is compatible with the interface condition (1) when $C_{t r} \equiv 4$. The numerical solution obtained after few iterations of the adaptive algorithm presented above is plotted in Figure 5 for $p=1$, together with the respective meshes.

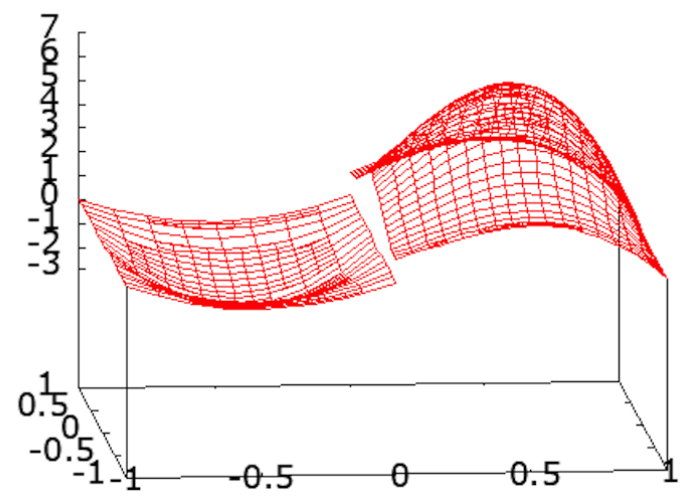

(a) 6th iteration

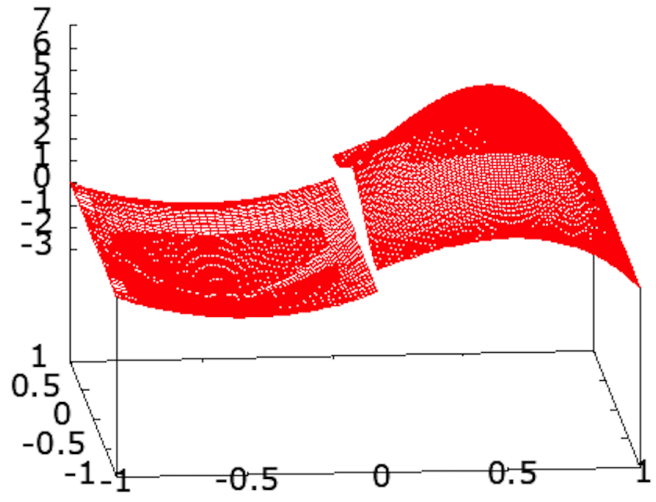

(b) 11th iteration

Figure 5: Example 1. Solution profiles.

The estimator and various norms of the error are plotted in Figure 6 under adaptive mesh refinement. Optimal rates of convergence are observed with respect to the degrees of freedom for both the estimator and the error measured in the dG-energy (and equivalent) norm(s). 


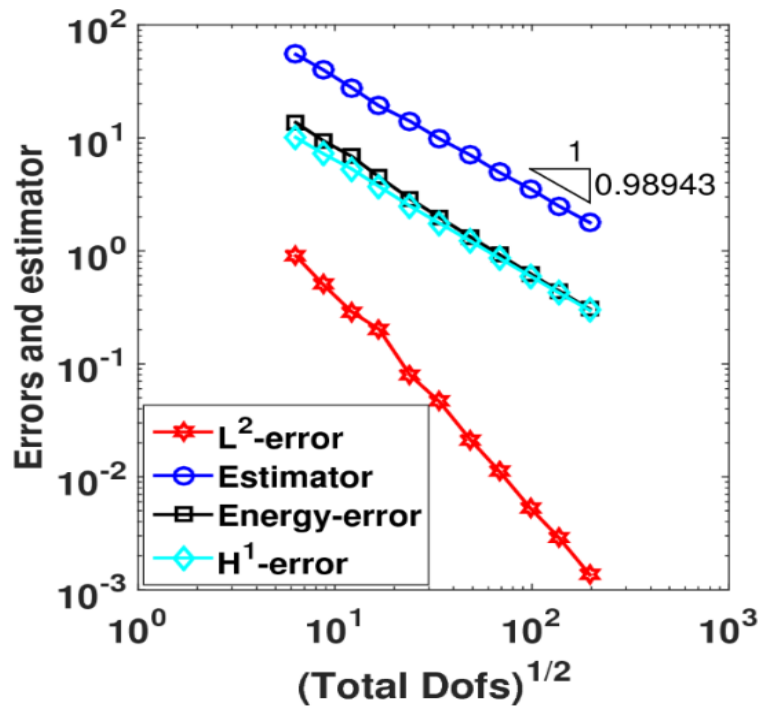

(a) $p=1$.

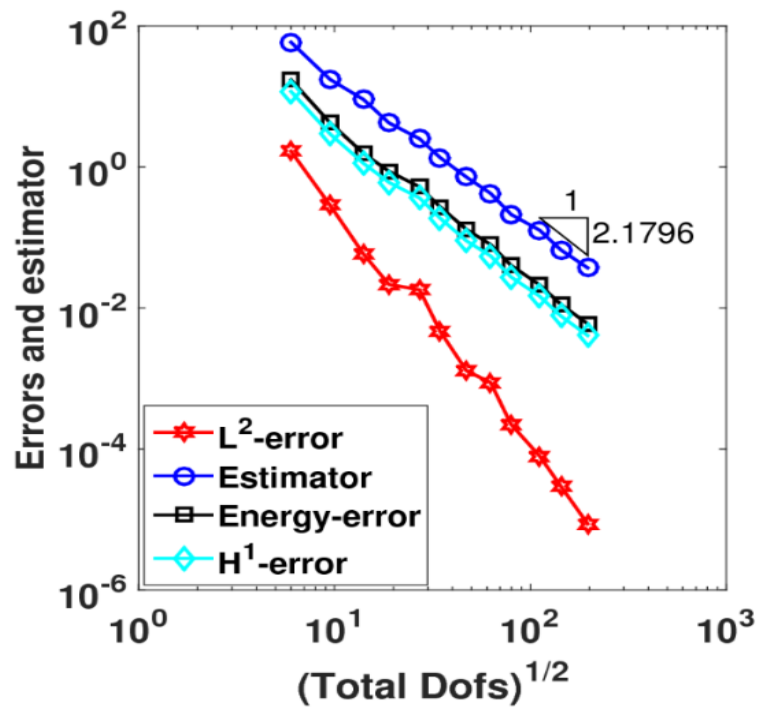

(b) $p=2$.

Figure 6: Example 1. Estimator and errors for adaptive mesh refinement for $p=1,2$.

\subsection{Example 2}

Let $\Omega=(-1,1)^{2} \backslash(0,1) \times(-1,0)$,subdivided into two subdomains interfacing at $x=0.125$. We consider the classical problem with $f=0$ and non-homogeneous Dirichlet boundary conditions compatible to the exact solution

$$
u=r^{2 / 3} \sin (2 \theta / 3),
$$

with $r=\sqrt{x^{2}+y^{2}}, \theta=\tan ^{-1}(y / x)$, and $C_{t r} \equiv 1$.

The purpose of this example is to observe the behaviour of the adaptive procedure based on the presented a posteriori error estimator, in presence of both a singularity (at the reentrant corner) and the jump discontinuity at the interface. The numerical solution obtained after six iterations of the adaptive algorithm is plotted in Figure 7. The refinement near the origin indicates that the error

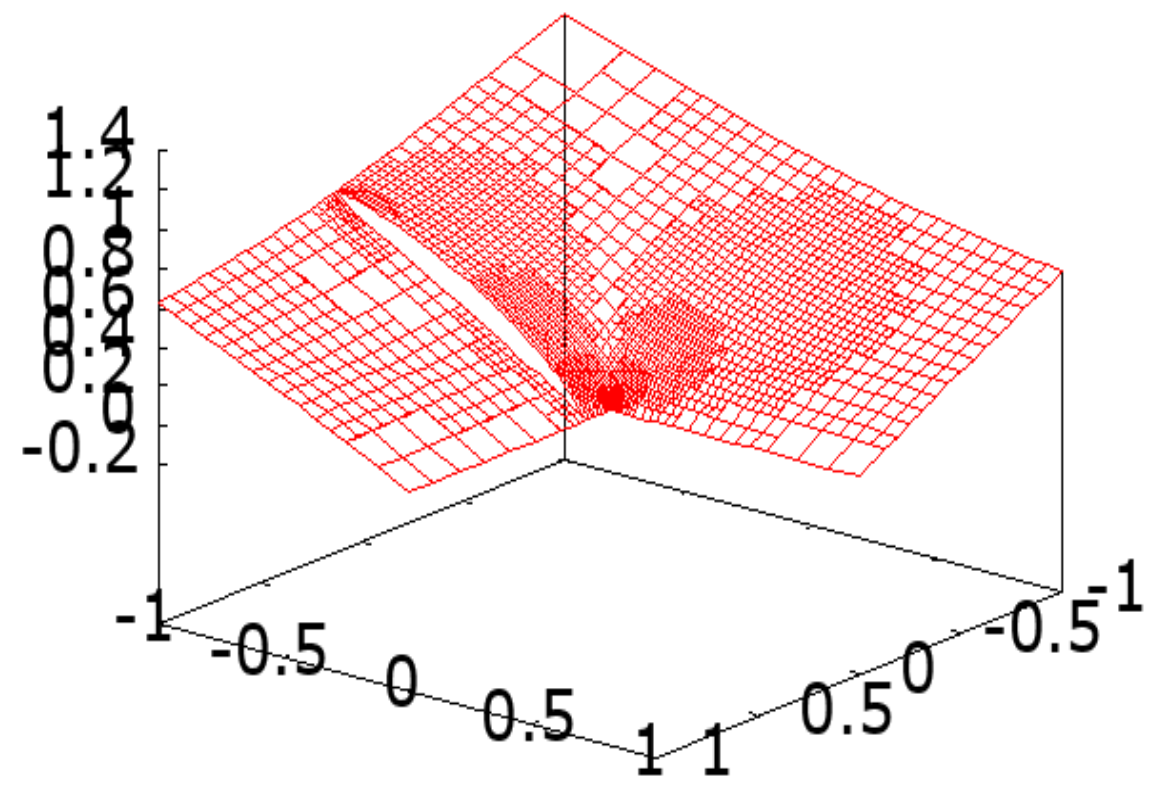

(a) 6th iteration

Figure 7: Example 2. Solution profile with $p=1$. 
estimator is practical and captures both the interface and singularity. The convergence plots reported in Figure 8 confirm that the estimator converges at the expected rates for both uniform and adaptive refinements.
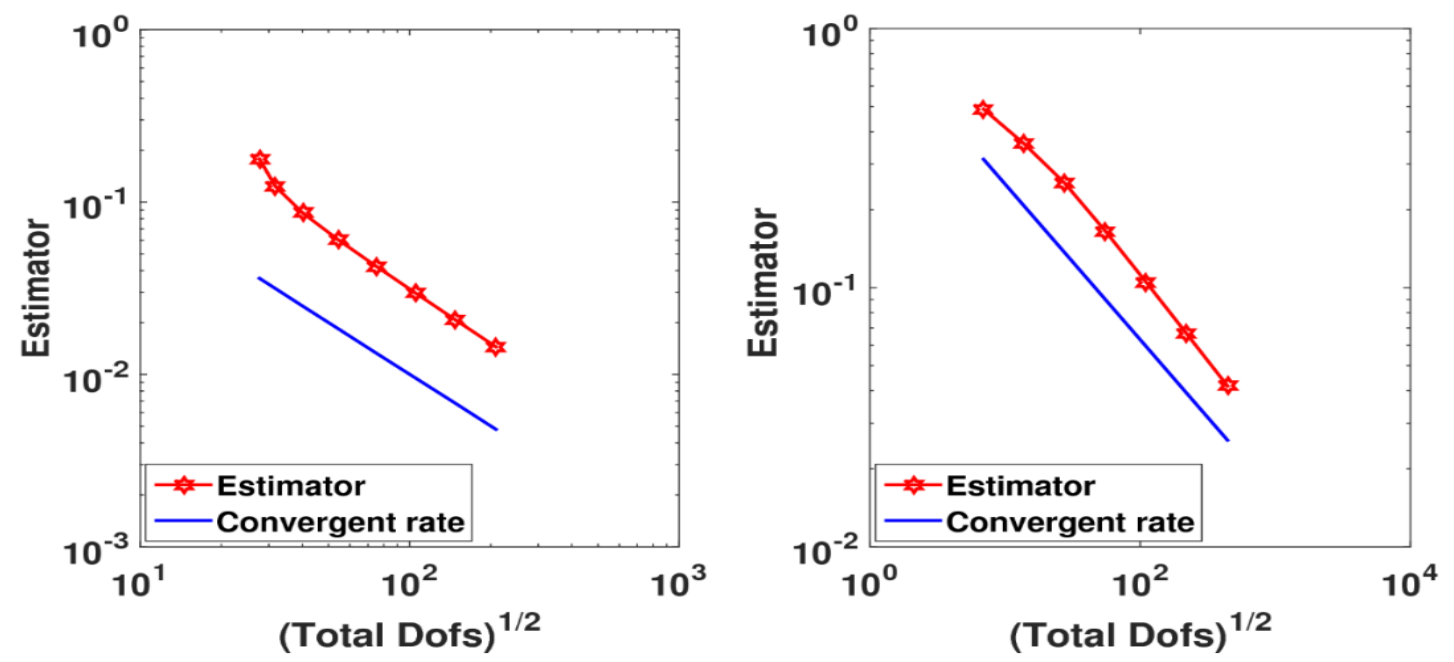

(a) $p=1$.
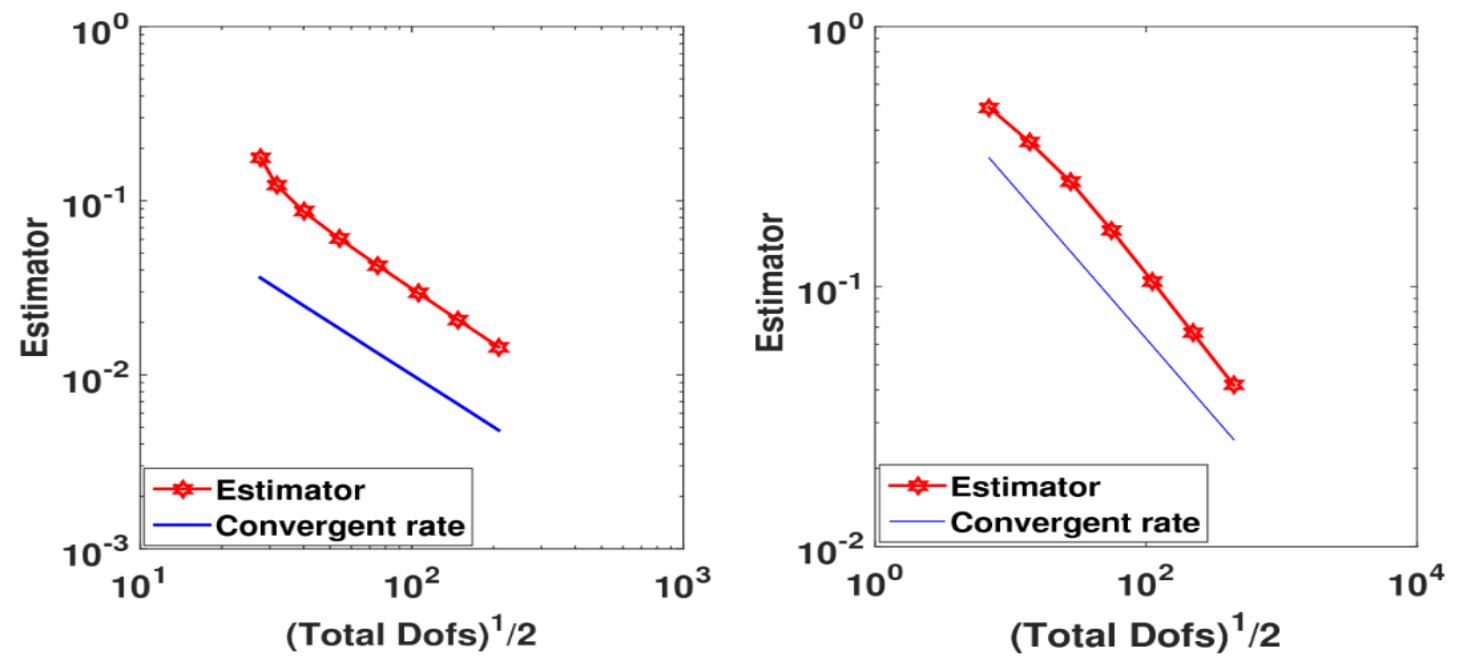

(b) $p=2$.

Figure 8: Example 2. Estimator and expected convergence rate for adaptive (left) and uniform (right) meshes for $p=1$ (above) and $p=2$ (below).

\section{Conclusions}

This work is devoted to the convergence analysis of adaptive discontinuous Galerkin methods for the numerical approximation of elliptic interface problems involving possibly curved interfaces and arising in the modelling of mass transfer of solutes through semi-permeable membranes. Adaptive algorithms were investigated, addressing the derivation of the necessary contraction property which leads to proof of convergence of standard adaptive procedures.

The mesh design for curved elements is not treated in standard mesh generators. This creates a number of practical issues, such as the representation of curves at the algorithmic level and, crucially for us, the refinement of curved elements, which remains largely open. We remark that, for the convergence result presented in Section 6, we assumed sufficient properties on the curved elements so that a "good" bisection refinement strategy is available which results to refined meshes with the same geometric properties for the curved elements. However, in all the numerical tests, we were not able to find triangulations violating such assumptions in practice. 
This work and the related results presented in [13] provide the basis for further development in the numerical solution of curved interface problems. The extension of the discontinuous Galerkin method to essentially arbitrarily-shaped elements, including curved elements not limited to the treatment of non-essential interface/boundary conditions, has been proposed very recently in [11]. This approach may be extended to tackle classical transmission/interface problems, see e.g., [19, 7], although the treatment of curved domains in an a posteriori fashion remains an open problem. Another interesting generalization of this work is the case of unfitted meshes [19], which are widely accepted as more practical, especially in the context of temporally moving interfaces.

[1] Agmon, S. (2010). Lectures on elliptic boundary value problems, volume 369. American Mathematical Soc.

[2] Bangerth, W., Hartmann, R., and Kanschat, G. (2007). deal.ii-a general-purpose object-oriented finite element library. ACM Transactions on Mathematical Software (TOMS), 33(4), 24.

[3] Becker, R., Burman, E., and Hansbo, P. (2011). A hierarchical NXFEM for fictitious domain simulations. Internat. J. Numer. Methods Engrg., 86(4-5), 549-559.

[4] Bonito, A. and Nochetto, R.H. (2010). Quasi-optimal convergence rate of an adaptive discontinuous galerkin method. SIAM Journal on Numerical Analysis, 48(2), 734-771.

[5] Burman, E. and Hansbo, P. (2010). Fictitious domain finite element methods using cut elements: I. A stabilized Lagrange multiplier method. Comput. Methods Appl. Mech. Engrg., 199(41-44), $2680-2686$.

[6] Burman, E. and Hansbo, P. (2010). Interior-penalty-stabilized Lagrange multiplier methods for the finite-element solution of elliptic interface problems. IMA J. Numer. Anal., 30(3), 870-885.

[7] Cai, Z., He, C., and Zhang, S. (2017). Discontinuous finite element methods for interface problems: Robust a priori and a posteriori error estimates. SIAM Journal on Numerical Analysis, 55(1), $400-418$.

[8] Cai, Z., Ye, X., and Zhang, S. (2011). Discontinuous Galerkin finite element methods for interface problems: A priori and a posteriori error estimations. SIAM Journal on Numerical Analysis, 49(5), 1761-1787.

[9] Calabrò, F. and Zunino, P. (2006). Analysis of parabolic problems on partitioned domains with nonlinear conditions at the interface. Application to mass transfer through semi-permeable membranes. Math. Models Methods Appl. Sci., 16(4), 479-501.

[10] Cangiani, A. and Natalini, R. (2010). A spatial model of cellular molecular trafficking including active transport along microtubules. J. Theoret. Biol., 267(4), 614-625.

[11] Cangiani, A., Dong, Z., and Georgoulis, E.H. (2019). $h p$-version version discontinuous galerkin methods on essentially arbitrarily-shaped elements. arXiv preprint arXiv:1906.01715.

[12] Cangiani, A., Georgoulis, E.H., and Jensen, M. (2013). Discontinuous Galerkin Methods for Mass Transfer through Semipermeable Membranes. SIAM J. Numer. Anal., 51(5), 2911-2934.

[13] Cangiani, A., Georgoulis, E.H., and Sabawi, Y. (2018). Adaptive discontinuous Galerkin method for elliptic interface problems. Math. Comp., 87, 2675-2707.

[14] Cascon, J.M., Kreuzer, C., Nochetto, R.H., and Siebert, K.G. (2008). Quasi-optimal convergence rate for an adaptive finite element method. SIAM Journal on Numerical Analysis, 46(5), 25242550 .

[15] Cascon, J.M., Kreuzer, C., Nochetto, R.H., and Siebert, K.G. (2008). Quasi-optimal convergence rate for an adaptive finite element method. SIAM J. Numer. Anal., 46(5), 2524-2550. 
[16] Dörfler, W. (1996). A convergent adaptive algorithm for Poisson's equation. SIAM J. Numer. Anal., 33(3), 1106-1124.

[17] Feng, X. and Karakashian, O.A. (2001). Two-level additive Schwarz methods for a discontinuous Galerkin approximation of second order elliptic problems. SIAM J. Numer. Anal., 39(4), 13431365 (electronic).

[18] Guzmán, J., Sánchez, M.A., and Sarkis, M. (2016). On the accuracy of finite element approximations to a class of interface problems. Math. Comp., 85(301), 2071-2098.

[19] Hansbo, A. and Hansbo, P. (2002). An unfitted finite element method, based on Nitsche's method, for elliptic interface problems. Comput. Methods Appl. Mech. Engrg., 191(47-48), 5537-5552.

[20] Hoppe, R.H.W., Kanschat, G., and Warburton, T. (2008/09). Convergence analysis of an adaptive interior penalty discontinuous Galerkin method. SIAM J. Numer. Anal., 47(1), 534-550.

[21] Hoppe, R.H.W. and Sharma, N. (2013). Convergence analysis of an adaptive interior penalty discontinuous Galerkin method for the Helmholtz equation. IMA J. Numer. Anal., 33(3), 898921.

[22] Karakashian, O.A. and Pascal, F. (2003). A posteriori error estimates for a discontinuous Galerkin approximation of second-order elliptic problems. SIAM J. Numer. Anal., 41(6), 2374-2399 (electronic).

[23] Karakashian, O.A. and Pascal, F. (2007). Convergence of adaptive discontinuous Galerkin approximations of second-order elliptic problems. SIAM J. Numer. Anal., 45(2), 641-665 (electronic).

[24] LeVeque, R.J. and Li, Z.L. (1994). The immersed interface method for elliptic equations with discontinuous coefficients and singular sources. SIAM J. Numer. Anal., 31(4), 1019-1044.

[25] Li, Z. (1998). The immersed interface method using a finite element formulation. Appl. Numer. Math., 27(3), 253-267.

[26] Massing, A., Larson, M.G., and Logg, A. (2013). Efficient implementation of finite element methods on nonmatching and overlapping meshes in three dimensions. SIAM J. Sci. Comput., $35(1), \mathrm{C} 23-\mathrm{C} 47$.

[27] Morin, P., Nochetto, R.H., and Siebert, K.G. (2000). Data oscillation and convergence of adaptive FEM. SIAM J. Numer. Anal., 38(2), 466-488 (electronic).

[28] Nicaise, S. and Cochez-Dhondt, S. (2010). Adaptive finite element methods for elliptic problems: abstract framework and applications. M2AN Math. Model. Numer. Anal., 44(3), 485-508.

[29] Peskin, C.S. (2002). The immersed boundary method. Acta Numer., 11, 479-517.

[30] Peterseim, D. (2014). Composite finite elements for elliptic interface problems. Math. Comp., 83(290), 2657-2674.

[31] Sun, H. and Darmofal, D.L. (2014). An adaptive simplex cut-cell method for high-order discontinuous Galerkin discretizations of elliptic interface problems and conjugate heat transfer problems. J. Comput. Phys., 278, 445-468.

[32] Zunino, P. (2009). Energy norm a-posteriori error estimates for a discontinuous Galerkin scheme applied to elliptic problems with an interface. In BAIL 2008-boundary and interior layers, volume 69 of Lect. Notes Comput. Sci. Eng., 295-304. Springer, Berlin. 